\title{
STOCHASTIC CALCULUS FOR \\ FRACTIONAL BROWNIAN MOTION \\ I. THEORY
}

\author{
Tyrone E. DUNCAN, Yaozhong HU and Bozenna PASIK-DUNCAN \\ Department of Mathematics \\ University of Kansas \\ 405 Snow Hall \\ Lawrence, KS 66045-2142
}

\section{Introduction}

Since the pioneering work of Hurst [14], [15], and Mandelbrot, [18], the fractional Brownian motions have played an increasingly important role in many fields of application such as hydrology, economics and telecommunications.

Let $0<H<1$. It is well known that there is a Gaussian stochastic process $\left(B_{t}^{H}, t \geq 0\right)$ such that

$$
\mathbb{E}\left(B_{t}^{H}\right)=0, \quad \mathbb{E}\left(B_{t}^{H} B_{s}^{H}\right)=\frac{1}{2}\left\{|t|^{2 H}+|s|^{2 H}-|t-s|^{2 H}\right\}
$$

for all $s, t \in \mathbb{R}_{+}$. This process is called a (standard) fractional Brownian motion with Hurst parameter $H$. To simplify the presentation, it is always assumed that the fractional Brownian motion is 0 at $t=0$.

If $H=1 / 2$, then the corresponding fractional Brownian motion is the usual standard Brownian motion. If $H>1 / 2$, then the process $\left(B_{t}^{H}, t \geq 0\right)$ exhibits a long-range dependence, that is, if $r(n)=\mathbb{E}\left[\left(B_{1}^{H}\left(B_{n+1}^{H}-B_{n}^{H}\right)\right]\right.$, then $\sum_{n=1}^{\infty} r(n)=\infty$. A fractional Brownian motion is also self-similar, that is, $\left(B_{\alpha t}^{H}, t \geq 0\right)$ has the same probability law as $\left(\alpha^{H} B_{t}^{H}, t \geq 0\right)$. A process satisfying this property is called a self-similar process with the Hurst parameter $H$.

\footnotetext{
${ }^{0}$ AMS 1991 subject classifications. Primary 60H05; Secondary 60H30, 60G15, 60 G18.

Keywords and phrases. Fractional Brownian motion, Itô integral, Stratonovich integral, Itô formula, Wick product, Itô calculus, multiple Itô integrals, multiple Stratonovich integral

Research supported partially by NSF Grant DMS 9623439
} 
Since in many problems related to network traffic analysis, mathematical finance and many other fields, the processes under study seem empirically to exhibit the self-similar properties and the long-range dependent properties and since the fractional Brownian motions are the simplest processes of this kind, it is important to have a systematic study of these processes and to use them to construct other stochastic processes. One way to approach this study is to follow, by analogy, the methods for Brownian motion. In the stochastic analysis, a Brownian motion can be used as the input (white) noise and many other processes (e.g. general diffusion processes) can be constructed as solutions of stochastic differential equations. One powerful tool for determining these solutions is the Itô formula.

However, it is also known that if a stochastic process $\left(\pi_{t}, t \geq 0\right)$ for which the stochastic integral $\int F_{t} d \pi_{t}$ is well defined for a large class of integrands $\left(F_{t}, t \geq 0\right)$, then this process $\left(\pi_{t}, t \geq 0\right)$ must be a semimartingale e.g. [20]. It is known that the fractional Brownian motions are not semimartingales. Therefore the beautiful classical theory of stochastic analysis [4] is not applicable to fractional Brownian motions for $H \neq 1 / 2$. It is a significant and challenging problem to extend the results in the classical stochastic analysis to these fractional Brownian motions. There have been a few papers in this direction: Lin [17] and Dai and Heyde [2] introduced stochastic integrals and extended the Itô formula to fractional Brownian motions. Their definitions of stochastic integral give a stochastic integral of Stratonovich type, which is explained further in Section 3. Their Itô formula is the usual chain rule for differentiation.

The stochastic integral $\int_{0}^{t} f_{s} \delta B_{s}^{H}$ with respect to the fractional Brownian motions, introduced by Lin, Dai and Heyde, does not satisfy the following property, $\mathbb{E} \int_{0}^{t} f_{s} \delta B_{s}^{H}=0$. A new type of stochastic integral $\int_{0}^{t} f_{s} d B_{s}^{H}$ is introduced satisfying $\mathbb{E} \int_{0}^{t} f_{s} d B_{s}^{H}=0$. This property seems to be important in the modeling problem by stochastic differential equations with fractional Gaussian noise as the driving random process. Consider the following type of differential equation

$$
d X_{t}=b\left(X_{t}\right) d t+\sigma\left(X_{s}\right) d B_{t}^{H} .
$$

It is natural to consider that $b\left(X_{t}\right)$ is the mean rate of change of the system state $X_{t}$ at time $t$ and $\sigma\left(X_{t}\right) d B_{t}^{H}$ is the random perturbation. So the term $\sigma\left(X_{t}\right) d B_{t}^{H}$ should not contribute to the mean rate of change. The term $b\left(X_{t}\right)$ is used to represent the average or deterministic part of the problem and $\sigma\left(X_{t}\right)$ is used to represent the intensity of the random part of the problem. Therefore it is important to extend the classical interpretation of $b$ and $\sigma$ to the differential equation (1.2).

To introduce the new integral $\int f d B^{H}$, the Wick product or Wick calculus is used. The use of Wick product is not anomalous because in white noise analysis the usual product has been associated with integrals of Stratonovich type and the Wick product has been associated with integrals of Itô type (e.g. [8], [12]).

A brief outline of the paper is given now. In Section 2, some description and terminology for the fractional Brownian motions are given. In Section 3, a derivative in special directions is defined and a stochastic integral of Itô type is defined using the Wick product. Furthermore, a stochastic integral of Stratonovich type is defined and the two types of stochastic integrals are related. In Section 4, some change of variables formulas (Itô for- 
mulas) are given for the two types of stochastic integrals. In Section 5, two applications of the Itô formula are given. In Section 6, multiple integrals of Itô type and Stratonovich type for a fractional Brownian motion are defined and the $\mathrm{Hu}-\mathrm{Meyer}$ formula is extended to these multiple integrals. The relation between these two types of multiple integrals is given. A square integrable functional of a fractional Brownian motion is represented as an infinite sum of orthogonal terms.

If the integral of a stochastic integral of Stratonovich type for a fractional Brownian motion with $H \in(1 / 2,1)$ has continuous sample paths, then the integral can be defined as a limit of a sequence of Riemann sums, where the integrand can be evaluated at any point between each pair of partition points. It is well known that this choice of evaluation of the integrand is not valid if the the integrator is Brownian motion, that is, $H=1 / 2$. If $f$ is smooth, then an application of an Itô formula is

$$
f\left(B_{t}^{H}\right)=f(0)+\int_{0}^{t} f^{\prime}\left(B_{s}^{H}\right) d B_{s}^{H}+H \int_{0}^{t} s^{2 H-1} f^{\prime \prime}\left(B_{s}^{H}\right) d s,
$$

where prime denotes differentiation and $H \in(1 / 2,1)$. It is interesting to note that if $H=1 / 2$ is formally substituted in the equation, then the well known Itô formula for Brownian motion is obtained.

\section{Fractional Brownian Motion}

Let $\Omega=C_{0}\left(\mathbb{R}_{+}, \mathbb{R}\right)$ be the space of real-valued continuous functions on $\mathbb{R}_{+}$with the initial value zero and the topology of local uniform convergence. There is a probability measure $P^{H}$ on $(\Omega, \mathcal{F})$, where $\mathcal{F}$ is the Borel $\sigma$-algebra such that on the probability space $\left(\Omega, \mathcal{F}, P^{H}\right)$ the coordinate process $B^{H}: \Omega \rightarrow \mathbb{R}$ defined as

$$
B_{t}^{H}(\omega)=\omega(t), \quad \omega \in \Omega
$$

is a Gaussian process satisfying (1.1). The process $\left(B_{t}^{H}, t \geq 0\right)$ is called the canonical (standard) fractional Brownian motion with Hurst parameter $H$. In this paper only this canonical process and its associated probability space are used. Throughout this paper it is assumed that $H \in\left(\frac{1}{2}, 1\right)$ is arbitrary but fixed. Clearly if $H=1 / 2$, the fractional Brownian motion is the standard Brownian motion.

It is elementary to verify that a fractional Brownian motion for $H \neq 1 / 2$ is not a semimartingale. It is known [20] that if the (usual) stochastic integral $\int_{a}^{b} f_{s} d X_{s}$ is well defined for a large family of integrands with respect to a process $\left(X_{t}, t \geq 0\right)$, then this process $\left(X_{t}, t \geq 0\right)$ is a semimartingale. Thus the well developed classical theory for semimartingales cannot be applied here, and the stochastic integral with respect to fractional Brownian motions needs to be developed.

Let $\phi: \mathbb{R}_{+} \times \mathbb{R}_{+} \rightarrow \mathbb{R}_{+}$be given by

$$
\phi(s, t)=H(2 H-1)|s-t|^{2 H-2} .
$$


Many results of this paper can be extended to a more general $\phi(s, t)$ that is symmetric and positive definite, so $\phi$ in (2.1) is given as a function of two variables and not their difference. Let $f: \mathbb{R}_{+} \rightarrow \mathbb{R}$ be a Borel measurable (deterministic) function. The function $f \in L_{\phi}^{2}\left(\mathbb{R}_{+}\right)$if

$$
|f|_{\phi}^{2}:=\int_{0}^{\infty} \int_{0}^{\infty} f(s) f(t) \phi(s, t) d s d t<\infty
$$

The inner product on the Hilbert space $L_{\phi}^{2}$ is denoted by $\langle\cdot, \cdot\rangle_{\phi}$.

The stochastic (Wiener) integral with respect to fractional Brownian motions for deterministic kernels is easily defined.

Lemma 2.1 If $f, g \in L_{\phi}^{2}\left(\mathbb{R}_{+}\right)$, then $\int_{0}^{\infty} f_{s} d B_{s}^{H}$ and $\int_{0}^{\infty} g_{s} d B_{s}^{H}$ are well defined zero mean, Gaussian random variables with variances $|f|_{\phi}^{2}$ and $|g|_{\phi}^{2}$ respectively and

$$
\mathbb{E}\left(\int_{0}^{\infty} f_{s} d B_{s}^{H} \int_{0}^{\infty} g_{s} d B_{t}^{H}\right)=\int_{0}^{\infty} \int_{0}^{\infty} f(s) g(t) \phi(s, t) d s d t=\langle f, g\rangle_{\phi} .
$$

This lemma is verified in [7]. It can be proved directly by verifying it for simple functions $\sum_{i=1}^{n} a_{i} \chi_{\left[t_{i}, t_{i+1}\right]}(s)$ and then proceeding with a passage to the limit.

\section{Stochastic Integration for Fractional Brownian Mo- tions}

Let $\left(\Omega, \mathcal{F}, P^{H}\right)$ be the probability space from Section 2 where a fractional Brownian motion with Hurst parameter $H$ is well defined. The probability measure $P^{H}$ depends on $H$. Throughout this paper the Hurst parameter $H$ is fixed such that $1 / 2<H<1$. Since $H$ is fixed, the probability measure is denoted by $P$.

Let $L^{p}(\Omega, \mathcal{F}, P)=L^{p}$ be the space of all random variables $F: \Omega \rightarrow \mathbb{R}$ such that

$$
\|F\|_{p}:=\left(\mathbb{E}|F|^{p}\right)^{1 / p}<\infty
$$

and let $L_{\phi}^{2}\left(\mathbb{R}_{+}\right)=\left\{\left.f\left|f: \mathbb{R}_{+} \rightarrow \mathbb{R},\right| f\right|_{\phi} ^{2}:=\int_{0}^{\infty} \int_{0}^{\infty} f_{s} f_{t} \phi(s, t) d s d t<\infty\right\}$. Often for notational simplicity $L_{\phi}^{2}\left(\mathbb{R}_{+}\right)$is denoted by $L_{\phi}^{2}$. For any $f \in L_{\phi}^{2}$, define $\varepsilon: L_{\phi}^{2} \rightarrow L^{1}(\Omega, \mathcal{F}, P)$ as

$$
\begin{aligned}
\varepsilon(f) & :=\exp \left\{\int_{0}^{\infty} f_{t} d B_{t}^{H}-\frac{1}{2} \int_{0}^{\infty} \int_{0}^{\infty} f_{s} f_{t} \phi(s, t) d s d t\right\} \\
& =\exp \left[\int_{0}^{\infty} f_{t} d B_{t}^{H}-\frac{1}{2}|f|_{\phi}^{2}\right] .
\end{aligned}
$$

If $f \in L_{\phi}^{2}$, then $\varepsilon(f) \in L^{p}(\Omega, \mathcal{F}, P)$ for each $p \geq 1$ and $\varepsilon(f)$ is called an exponential function (e.g. [21]). The Hilbert space $L_{\phi}^{2}$ is naturally associated with the Gaussian process, fractional Brownian motion, by the approach using an abstract Wiener space. The Hilbert space plays a basic role for questions of absolute continuity [6] and the exponential function (3.1) is a Radon-Nikodym derivative for a translate of the fractional Brownian motion. 
Let $\mathcal{E}$ be the linear span of the exponentials, that is,

$$
\mathcal{E}=\left\{\sum_{k=1}^{n} a_{k} \varepsilon\left(f_{k}\right), \quad n \in \mathbb{N}, a_{k} \in \mathbb{R}, f_{k} \in L_{\phi}^{2}\left(\mathbb{R}_{+}\right) \text {for } k \in\{1, \cdots, n\}\right\} .
$$

Theorem $3.1 \mathcal{E}$ is a dense set of $L^{p}(\Omega, \mathcal{F}, P)$ for each $p \geq 1$. In particular, $\mathcal{E}$ is a dense set of $L^{2}(\Omega, \mathcal{F}, P)$.

Proof A functional $F: \Omega \rightarrow \mathbb{R}$ is said to be a polynomial of the fractional Brownian motion if there is a polynomial $p\left(x_{1}, x_{2}, \cdots, x_{n}\right)$ such that

$$
F=p\left(B_{t_{1}}^{H}, B_{t_{2}}^{H}, \cdots, B_{t_{n}}^{H}\right)
$$

for some $0 \leq t_{1}<t_{2}<\cdots<t_{n}$. Since $\left(B_{t}^{H}, t \geq 0\right)$ is a Gaussian process it is well known that the set of all polynomial fractional Brownian functionals is dense in $L^{p}(\Omega, \mathcal{F}, P)$ for $p \geq 1$. In this case, the denseness of the polynomials follows from the continuity of the process and the Stone-Weierstrass theorem. To prove the theorem it is only necessary to prove that any polynomial can be approximated by the elements in $\mathcal{E}$. Since the Wick product of exponentials is still an exponential it is easy to see that it is only necessary to show that for any $t>0, B_{t}^{H}$ can be approximated by elements in $\mathcal{E}$.

Let $f_{\delta}(s)=\chi_{[0, t]}(s) \delta$. It is clear that for $\delta>0, f_{\delta}$ is in $L_{\phi}^{2}$. Then $\varepsilon\left(f_{\delta}\right)=c(\delta) e^{\delta B_{t}^{H}}$ for some positive constant $c(\delta)$. It is easy to see that

$$
F_{\delta}=\frac{\varepsilon\left(f_{\delta}\right)-c(\delta)}{c(\delta) \delta}=\frac{e^{\delta B_{t}^{H}}-1}{\delta}
$$

is in $\mathcal{E}$. If $\delta \rightarrow 0$, then $F_{\delta} \rightarrow B_{t}^{H}$ in $L^{p}(\Omega, \mathcal{F}, P)$ for each $p \geq 1$. This completes the proof.

Theorem 3.2 If $f_{1}, f_{2}, \cdots, f_{n}$ are elements in $L_{\phi}^{2}$ such that $\left|f_{i}-f_{j}\right|_{\phi} \neq 0$ for $i \neq j$, then $\varepsilon\left(f_{1}\right), \varepsilon\left(f_{2}\right), \cdots, \varepsilon\left(f_{n}\right)$ are linearly independent in $L_{\phi}^{2}$.

Proof This theorem is known to be true if the fractional Brownian motion is replaced by a standard Brownian motion, (e.g. [21]).

Let $f_{1}, f_{2}, \cdots, f_{k}$ be distinct elements in $L_{\phi}^{2}$. Let $\lambda_{1}, \lambda_{2}, \cdots, \lambda_{k}$ be real numbers such that

$$
\left|\lambda_{1} \varepsilon\left(f_{1}\right)+\lambda_{2} \varepsilon\left(f_{2}\right)+\cdots+\lambda_{k} \varepsilon\left(f_{k}\right)\right|_{\phi}=0
$$

Thus for any $g \in L_{\phi}^{2}$,

$$
\mathbb{E}\left[\left\{\lambda_{1} \varepsilon\left(f_{1}\right)+\lambda_{2} \varepsilon\left(f_{2}\right)+\cdots+\lambda_{k} \varepsilon\left(f_{k}\right)\right\} \varepsilon(g)\right]=0 .
$$

By an elementary computation for Gaussian random variables it follows that

$$
\lambda_{1} e^{\left\langle f_{1}, g\right\rangle_{\phi}}+\lambda_{2} e^{\left\langle f_{2}, g\right\rangle_{\phi}}+\cdots+\lambda_{k} e^{\left\langle f_{k}, g\right\rangle_{\phi}}=0
$$


Replace $g$ by $\delta g$ for $\delta \in \mathbb{R}$ to obtain

$$
\lambda_{1} e^{\delta\left\langle f_{1}, g\right\rangle_{\phi}}+\lambda_{2} e^{\delta\left\langle f_{2}, g\right\rangle_{\phi}}+\cdots+\lambda_{k} e^{\delta\left\langle f_{k}, g\right\rangle_{\phi}}=0 .
$$

Expand the above identity in the powers of $\delta$ and compare the coefficients of $\delta^{p}$, for $p \in$ $\{0,1, \cdots, k-1\}$ to obtain the family of equations

$$
\lambda_{1}\left\langle f_{1}, g\right\rangle_{\phi}^{p}+\lambda_{2}\left\langle f_{2}, g\right\rangle_{\phi}^{p}+\cdots+\lambda_{k}\left\langle f_{k}, g\right\rangle_{\phi}^{p}=0
$$

for $p=0,1, \cdots, k-1$. This is a linear system of $k$ equations and $k$ unknowns. By the Vandermonde formula, the determinant of this linear system is

$$
\operatorname{det}\left(\left\langle f_{i}, g\right\rangle_{\phi}^{p}\right)=\prod_{i<j}\left\langle f_{i}-f_{j}, g\right\rangle_{\phi}^{p}
$$

For every pair $(i, j)$ with $i \neq j$, the set $\left\{g \in L_{\phi}^{2}:\left\langle f_{i}-f_{j}, g\right\rangle_{\phi} \neq 0\right\}$ is the complement of a hyperplane in $L_{\phi}^{2}$. Since the intersection of finitely many complements of hyperplanes in $L_{\phi}^{2}$ is not empty, there is a $g \in L_{\phi}^{2}$ such that $\left\langle f_{i}-f_{j}, g\right\rangle_{\phi} \neq 0$ for all pairs $i$ and $j$ such that $i \neq j$. Thus $\lambda_{1}=\lambda_{2} \cdots=\lambda_{k}=0$. This proves the theorem .

The above two theorems reduce many verifications for functions in $L^{2}(\Omega, \mathcal{F}, P)$ to verifications of exponentials in $\mathcal{E}$.

The following result is an absolute continuity of measures for some translates of fractional Brownian motion.

Theorem 3.3 If $F: \Omega \rightarrow \mathbb{R}$ is a random variable such that $F \in L^{p}(\Omega, \mathcal{F}, P)$ for some $p \geq 1$, then

$$
\mathbb{E}\left\{F\left(B_{\cdot}^{H}+\int_{0}(\Phi g)(s) d s\right)\right\}=\mathbb{E}\left\{F\left(B_{.}^{H}\right) e^{\int_{0}^{\infty} g_{s} d B_{s}^{H}-\frac{1}{2} \int_{0}^{\infty} \int_{0}^{\infty} \phi(u, v) g_{u} g_{v} d u d v}\right\}
$$

where $\Phi$ is given by

$$
(\Phi g)(t)=\int_{0}^{\infty} \phi(t, u) g_{u} d u
$$

and $g \in L_{\phi}^{2}$.

Proof The term $F\left(B^{H}\right)$ denotes $F(\omega)$. Let $k \in L_{\phi}^{2}$ and

$$
F\left(B^{H}\right)=\varepsilon(k)=e^{\int_{0}^{\infty} k_{s} d B_{s}^{H}-\frac{1}{2} \int_{0}^{\infty} \int_{0}^{\infty} \phi(u, v) k_{u} k_{v} d u d v} .
$$

Then

$$
F\left(B_{.}^{H}+\int_{0}^{\cdot}(\Phi g)_{s} d s\right)=F\left(B_{\cdot}^{H}\right) e^{\int_{0}^{\infty} k_{u}(\Phi g)_{u} d u} .
$$

So

$$
\mathbb{E}\left\{F\left(B_{\cdot}^{H}+\int_{0}^{\cdot}(\Phi g)_{s} d s\right)\right\}=e^{\int_{0}^{\infty} k_{u}(\Phi g)_{u} d u}
$$


Furthermore it follows that

$$
\begin{aligned}
\mathbb{E}\left\{F\left(B_{\cdot}^{H}\right) \varepsilon(g)\right\} & =e^{\int_{0}^{\infty} \int_{0}^{\infty} \phi(u, v) k_{u} g_{v} d u d v} \\
& =e^{\int_{0}^{\infty} k_{u}(\Phi g)_{u} d u}
\end{aligned}
$$

Thus the theorem is true if $F$ is an exponential function $\varepsilon(f) \in \mathcal{E}$. A limiting argument completes the proof.

Let a Radon-Nikodym derivative $\frac{d \tilde{P}}{d P}$ on $(\Omega, \mathcal{F}, P)$ be given by

$$
\frac{d \tilde{P}}{d P}=e^{\int_{0}^{\infty} g_{s} d B_{s}^{H}-\frac{1}{2} \int_{0}^{\infty} \int_{0}^{\infty} \phi(u, v) g_{u} g_{v} d u d v}
$$

and denote the expectation with respect to $\tilde{P}$ by $\tilde{\mathbb{E}}$, then $(3.3)$ is given by

$$
\mathbb{E} F\left(B_{\cdot}^{H}+\int_{0}^{\cdot}(\Phi g)_{s} d s\right)=\tilde{\mathbb{E}}\left(F\left(B_{\cdot}^{H}\right)\right) .
$$

For a functional $F$ in $L^{p}(\Omega, \mathcal{F}, P)(p \geq 1)$ and a function $g \in L_{\phi}^{2}, F\left(B^{H}+\int_{0}^{\cdot}(\Phi g)(v) d v\right)$ is well defined. An analogue of the Malliavin derivative [23] is introduced.

Definition 3.1 The $\phi$-derivative of a random variable $F \in L^{p}$ in the direction of $\Phi g$ is defined as

$$
D_{\Phi g} F(\omega)=\lim _{\delta \rightarrow 0} \frac{1}{\delta}\left\{F\left(\omega+\delta \int_{0}^{\cdot}(\Phi g)(u) d u\right)-F(\omega)\right\}
$$

if the limit exists in $L^{p}(\Omega, \mathcal{F}, P)$. Furthermore if there is a process $\left(D^{\phi} F_{s}, s \geq 0\right)$ such that

$$
D_{\Phi g} F=\int_{0}^{\infty} D^{\phi} F_{s} g_{s} d s \quad \text { a.s. }
$$

for all $g \in L_{\phi}^{2}$ then $F$ is said to be $\phi$-differentiable.

The higher order derivatives can be defined in a similar manner.

Definition 3.2 Let $F:[0, T] \times \Omega \rightarrow \mathbb{R}$ be a stochastic process. The process $F$ is said to be $\phi$-differentiable if for each $t \in[0, T], F(t, \cdot)$ is $\phi$-differentiable and $D_{s}^{\phi} F_{t}$ is jointly measurable.

It is easy to verify an elementary version of a chain rule, that is, if $f: \mathbb{R} \rightarrow \mathbb{R}$ is a smooth function and $F: \Omega \rightarrow \mathbb{R}$ is $\phi$-differentiable then $f(F)$ is also $\phi$-differentiable and

$$
D_{\Phi g} f(F)=f^{\prime}(F) D_{\Phi g} F
$$

and

$$
D_{s}^{\phi} f(F)=f^{\prime}(F) D_{s}^{\phi} F
$$


and the iterated directional derivatives

$$
D_{\Phi g_{1}} D_{\Phi g_{2}} f(F)=f^{\prime}(F) D_{\Phi g_{1}} D_{\Phi g_{2}} F+f^{\prime \prime}(F) D_{\Phi g_{1}} F D_{\Phi g_{2}} F
$$

The following rules for differentiation which can be verified as in the proof of Proposition 3.4 are useful later

$$
\begin{gathered}
D_{\Phi g} \int_{0}^{\infty} f_{s} d B_{s}^{H}=\int_{0}^{\infty} \int_{0}^{\infty} \phi(u, v) f_{u} g_{v} d u d v=\langle f, g\rangle_{\phi} \\
D_{s}^{\phi} \int_{0}^{\infty} f_{u} d B_{u}^{H}=\int_{0}^{\infty} \phi(u, s) f_{u} d u=(\Phi f)(s) ; \\
D_{\Phi g} \varepsilon(f)=\varepsilon(f) \int_{0}^{\infty} \int_{0}^{\infty} \phi(u, v) f_{u} g_{v} d u d v=\varepsilon(f)\langle f, g\rangle_{\phi} ; \\
D_{s}^{\phi} \varepsilon(f)=\varepsilon(f) \int_{0}^{\infty} \phi(u, s) f_{u} d u=\varepsilon(f)(\Phi f)(s),
\end{gathered}
$$

where $f, g \in L_{\phi}^{2}$.

Now the Wick product $\diamond$ of two functionals is introduced. To extend the theory of stochastic calculus for Brownian motions to the fractional Brownian motions, the Wick calculus for Gaussian processes (or Gaussian measures) is used. The Wick product of two exponentials $\varepsilon(f)$ and $\varepsilon(g)$ is defined as

$$
\varepsilon(f) \diamond \varepsilon(g)=\varepsilon(f+g) .
$$

Since for distinct $f_{1}, f_{2}, \cdots, f_{n}$ in $L_{\phi}^{2}, \varepsilon\left(f_{1}\right), \varepsilon\left(f_{2}\right), \cdots, \varepsilon\left(f_{n}\right)$ are linearly independent, this definition can be extended to define the Wick product $F \diamond G$ of two functionals $F$ and $G$ in $\mathcal{E}$.

Note that $\int_{0}^{\infty} g_{s} d B_{s}^{H}$ is not an element in $\mathcal{E}$. The Wick product is extended to more general functionals, including the functionals of the form $\int_{0}^{\infty} g_{s} d B_{s}^{H}$, where $g \in L_{\phi}^{2}$.

Proposition 3.4 If $g \in L_{\phi}^{2}, F \in L^{2}(\Omega, \mathcal{F}, P)$ and $D_{\Phi g} F \in L^{2}(\Omega, \mathcal{F}, P)$, then

$$
F \diamond \int_{0}^{\infty} g_{s} d B_{s}^{H}=F \int_{0}^{\infty} g_{s} d B_{s}^{H}-D_{\Phi g} F
$$

Proof By the definition (3.9),

$$
\varepsilon(f) \diamond \varepsilon(\delta g)=\varepsilon(f+\delta g)
$$

Differentiate the above identity with respect to $\delta$ and evaluate at $\delta=0$, to obtain

$$
\begin{aligned}
\varepsilon(f) \diamond \int_{0}^{\infty} g_{s} d B_{s}^{H} & =\varepsilon(f)\left[\int_{0}^{\infty} g_{s} d B_{s}^{H}-\langle f, g\rangle_{\phi}\right] \\
& =\varepsilon(f) \int_{0}^{\infty} g_{s} d B_{s}^{H}-\varepsilon(f)\langle f, g\rangle_{\phi}
\end{aligned}
$$


By (3.7) it follows that the last term of the above expression is $D_{\Phi g} \varepsilon(f)$. Thus the following equality is satisfied

$$
\varepsilon(f) \diamond \int_{0}^{\infty} g_{s} d B_{s}^{H}=\varepsilon(f) \int_{0}^{\infty} g_{s} d B_{s}^{H}-D_{\Phi g} \varepsilon(f) .
$$

If $F \in \mathcal{E}$ is a finite linear combination of $\varepsilon\left(f_{1}\right), \varepsilon\left(f_{2}\right), \cdots, \varepsilon\left(f_{n}\right)$, then extend (3.13) by linearity

$$
\begin{aligned}
F \diamond \int_{0}^{\infty} g_{s} d B_{s}^{H} & =F \int_{0}^{\infty} g_{s} d B_{s}^{H}-D_{\Phi g} F \\
& =F \int_{0}^{\infty} g_{s} d B_{s}^{H}-\int_{0}^{\infty} D_{s}^{\phi} F g_{s} d s
\end{aligned}
$$

The proof of the proposition is completed by Theorem 3.1.

Now the second moment of (3.10) is computed. Note that by a simple computation for Gaussian random variables, it follows that

$$
\mathbb{E}(\varepsilon(f) \varepsilon(g))=\exp \left\{\langle f, g\rangle_{\phi}\right\}
$$

Thus

$$
\begin{aligned}
\mathbb{E}\{(\varepsilon(f) \diamond \varepsilon(\gamma g))(\varepsilon(h) \diamond \varepsilon(\delta g))\} & =\mathbb{E}\{\varepsilon(f+\gamma g) \varepsilon(h+\delta g)\} \\
& =\exp \left\{\langle f+\gamma g, h+\delta g\rangle_{\phi}\right\} .
\end{aligned}
$$

Both sides of this equality are functions of $\gamma$ and $\delta$. Taking the partial derivative $\frac{\partial^{2}}{\partial \gamma \partial \delta}$ evaluated at $\gamma=\delta=0$, it follows that

$$
\begin{aligned}
& \mathbb{E}\left\{\left(\varepsilon(f) \diamond \int_{0}^{\infty} g_{s} d B_{s}^{H}\right)\left(\varepsilon(h) \diamond \int_{0}^{\infty} g_{s} d B_{s}^{H}\right)\right\} \\
= & \exp \left(\langle f, h\rangle_{\phi}\right)\left\{\langle f, g\rangle_{\phi}\langle h, g\rangle_{\phi}+\langle g, g\rangle_{\phi}\right\} \\
= & \mathbb{E}\left\{D_{\Phi g} \varepsilon(f) D_{\Phi g} \varepsilon(h)+\varepsilon(f) \varepsilon(h)\langle g, g\rangle_{\phi}\right\}
\end{aligned}
$$

Thus

$$
\begin{aligned}
\mathbb{E}\left\{\left(\varepsilon(f) \diamond \int_{0}^{\infty} g_{s} d B_{s}^{H}\right)\left(\varepsilon(h) \diamond \int_{0}^{\infty} g_{s} d B_{s}^{H}\right)\right\}= & \mathbb{E}\left(D_{\Phi g} \varepsilon(f) D_{\Phi g} \varepsilon(h)\right. \\
& \left.+\varepsilon(f) \varepsilon(h)\langle g, g\rangle_{\phi}\right) .
\end{aligned}
$$

By bilinearity, for any $F$ and $G$ in $\mathcal{E}$, the following equality is satisfied

$$
\mathbb{E}\left\{\left(F \diamond \int_{0}^{\infty} g_{s} d B_{s}^{H}\right)\left(G \diamond \int_{0}^{\infty} g_{s} d B_{s}^{H}\right)\right\}=\mathbb{E}\left\{D_{\Phi g} F D_{\Phi g} G+F G\langle g, g\rangle_{\phi}\right\} .
$$

Let $F$ be equal to $G$. Then

$$
\mathbb{E}\left(F \diamond \int_{0}^{\infty} g_{s} d B_{s}^{H}\right)^{2}=\mathbb{E}\left[\left(D_{\Phi g} F\right)^{2}+F^{2}|g|_{\phi}^{2}\right] .
$$

This result is stated in the following theorem. 
Theorem 3.5 Let $g \in L_{\phi}^{2}$ and let $\mathcal{E}_{g}$ be the completion of $\mathcal{E}$ under the norm

$$
\|F\|_{g}^{2}=\mathbb{E}\left\{\left(D_{\Phi g} F\right)^{2}+F^{2}\right\}
$$

where $F$ is a random variable. Then for any element $F \in \mathcal{E}_{g}, F \diamond \int_{0}^{\infty} g_{s} d B_{s}^{H}$ is well defined and

$$
\mathbb{E}\left(F \diamond \int_{0}^{\infty} g_{s} d B_{s}^{H}\right)^{2}=\mathbb{E}\left\{\left(D_{\Phi g} F\right)^{2}+F^{2}|g|_{\phi}^{2}\right\}
$$

By the polarization technique [21], there is the following corollary.

Corollary 3.6 Let $g, h \in L_{\phi}^{2}$ and $F, G \in \mathcal{E}$. Then

$$
\mathbb{E}\left(F \diamond \int_{0}^{\infty} g_{s} d B_{s}^{H} G \diamond \int_{0}^{\infty} h_{s} d B_{s}^{H}\right)=\mathbb{E}\left[D_{\Phi g} F D_{\Phi h} G+F G\langle g, h\rangle_{\phi}\right] .
$$

This equality is the starting point for the definition of the stochastic integral with respect to the fractional Brownian motions. Let $F \in \mathcal{E}$. The stochastic integral $\int_{0}^{T} F_{s} d B_{s}^{H}$ is defined and some properties associated with this stochastic integral are studied.

Consider an arbitrary partition of $[0, T], \pi: 0=t_{0}<t_{1}<t_{1}<\cdots<t_{n}=T$. First the following Riemann sum is given using the Wick product introduced above

$$
S(F, \pi)=\sum_{i=0}^{n-1} F_{t_{i}} \diamond\left(B_{t_{i+1}}^{H}-B_{t_{i}}^{H}\right)
$$

From (3.9), it easily follows that that for any $F$ and $G$ in $\mathcal{E}, \mathbb{E}(F \diamond G)=\mathbb{E}(F) \mathbb{E}(G)$. This identity extends to more general $F$ and $G$ such that $F \diamond G$ is well defined (e.g. [8], p.83). Thus for any partition $\pi$,

$$
\begin{aligned}
\mathbb{E}\left(\sum_{i=0}^{n-1} F_{t_{i}} \diamond\left(B_{t_{i+1}}^{H}-B_{t_{i}}^{H}\right)\right) & =\sum_{i=0}^{n-1} \mathbb{E}\left(F_{t_{i}} \diamond\left(B_{t_{i+1}}^{H}-B_{t_{i}}^{H}\right)\right) \\
& =\sum_{i=0}^{n-1} \mathbb{E}\left(F_{t_{i}}\right) \mathbb{E}\left(B_{t_{i+1}}^{H}-B_{t_{i}}^{H}\right)=0
\end{aligned}
$$

To compute the $L^{2}$ norm of $S(F, \pi)$, denote

$$
\sigma_{i j}=\mathbb{E}\left\{\left(F_{t_{i}} \diamond\left(B_{t_{i+1}}^{H}-B_{t_{i}}^{H}\right)\right)\left(F_{t_{j}} \diamond\left(B_{t_{j+1}}^{H}-B_{t_{j}}^{H}\right)\right)\right\} .
$$

By Corollary 3.6, it follows that

$$
\sigma_{i j}=\mathbb{E}\left\{\int_{t_{i}}^{t_{i+1}} D_{s}^{\phi} F_{t_{i}} d s \int_{t_{j}}^{t_{j+1}} D_{t}^{\phi} F_{t_{j}} d t+F_{t_{i}} F_{t_{j}} \int_{t_{i}}^{t_{i+1}} \int_{t_{j}}^{t_{j+1}} \phi(u, v) d u d v\right\}
$$


Thus

$$
\mathbb{E} S(F, \pi)^{2}=\sum_{i, j=0}^{n-1} \mathbb{E}\left\{\int_{t_{i}}^{t_{i+1}} D_{s}^{\phi} F_{t_{i}} d s \int_{t_{j}}^{t_{j+1}} D_{t}^{\phi} F_{t_{j}} d t+F_{t_{i}} F_{t_{j}} \int_{t_{i}}^{t_{i+1}} \int_{t_{j}}^{t_{j+1}} \phi(u, v) d u d v\right\}
$$

Denote $|\pi|:=\max _{i}\left(t_{i+1}-t_{i}\right)$ and $F_{t}^{\pi}=F_{t_{i}}$ if $t_{i} \leq t<t_{i+1}$. Assume that as $|\pi| \rightarrow 0$, $\mathbb{E}\left|F^{\pi}-F\right|_{\phi}^{2} \rightarrow 0$ and

$$
\sum_{i=0}^{n-1} \mathbb{E}\left\{\int_{t_{i}}^{t_{i+1}}\left|D_{s}^{\phi} F_{t_{i}}-D_{s}^{\phi} F_{s}\right| d s\right\}^{2}
$$

converges to 0 . Then from the above it is easy to see that if $\left(\pi_{n}, n \in \mathbb{N}\right)$ is a sequence of partitions such that $\left|\pi_{n}\right| \rightarrow 0$ as $n \rightarrow \infty$, then $\left(S\left(F, \pi_{n}\right), n \in \mathbb{N}\right)$ is a Cauchy sequence in $L^{2}(\Omega, \mathcal{F}, P)$. The limit of this sequence in $L^{2}(\Omega, \mathcal{F}, P)$ is defined as $\int_{0}^{T} F_{s} d B_{s}^{H}:$ that is, define

$$
\int_{0}^{T} F_{s} d B_{s}^{H}=\lim _{|\pi| \rightarrow 0} \sum_{i=0}^{n-1} F_{t_{i}}^{\pi} \diamond\left(B_{t_{i+1}}^{H}-B_{t_{i}}^{H}\right)
$$

so that

$$
\mathbb{E}\left|\int_{0}^{T} F_{s} d B_{s}^{H}\right|^{2}=\mathbb{E}\left\{\left(\int_{0}^{T} D_{s}^{\phi} F_{s} d s\right)^{2}+|F|_{\phi}^{2}\right\} .
$$

Let $\mathcal{L}(0, T)$ be the family of stochastic processes on $[0, T]$ such that $F \in \mathcal{L}(0, T)$ if $\mathbb{E}|F|_{\phi}^{2}<\infty, F$ is $\phi$-differentiable, the trace of $\left(D_{s}^{\phi} F_{t}, 0 \leq s \leq T, 0 \leq t \leq T\right)$ exists and $\mathbb{E} \int_{0}^{T}\left(D_{s}^{\phi} F_{s}\right)^{2} d s<\infty$ and for each sequence of partitions $\left(\pi_{n}, n \in \mathbb{N}\right)$ such that $\left|\pi_{n}\right| \rightarrow 0$ as $n \rightarrow \infty$

$$
\sum_{i=0}^{n-1} \mathbb{E}\left\{\int_{t_{i}^{(n)}}^{t_{i+1}^{(n)}}\left|D_{s}^{\phi} F_{\left.t_{i}^{(} n\right)}^{\pi}-D_{s}^{\phi} F_{s}\right| d s\right\}^{2}
$$

and

$$
\mathbb{E}\left|F^{\pi}-F\right|_{\phi}^{2}
$$

tend to 0 as $n \rightarrow \infty$, where $\pi_{n}: 0=t_{0}^{(n)}<t_{1}^{(n)}<\cdots<t_{n-1}^{(n)}<t_{n}^{(n)}=T$.

The following result summarizes the above construction of a stochastic integral.

Theorem 3.7 Let $\left(F_{t}, t \in[0, T]\right)$ be a stochastic process such that $F \in \mathcal{L}(0, T)$. The limit (3.16) exists and this limit is defined as $\int_{0}^{T} F_{s} d B_{s}^{H}$. Moreover, this integral satisfies $\mathbb{E} \int_{0}^{T} F_{s} d B_{s}^{H}=0$ and

$$
\mathbb{E}\left|\int_{0}^{T} F_{s} d B_{s}^{H}\right|^{2}=\mathbb{E}\left\{\left(\int_{0}^{T} D_{s}^{\phi} F_{s} d s\right)^{2}+\left|1_{[0, T]} F\right|_{\phi}^{2}\right\} .
$$

The following properties follow directly from the above theorem.

1) If $F, G \in \mathcal{L}(0, T)$, then

$$
\int_{0}^{t}\left(a F_{s}+b G_{s}\right) d B_{s}^{H}=a \int_{0}^{t} F_{s} d B_{s}^{H}+b \int_{0}^{t} G_{s} d B_{s}^{H} \quad \text { a.s. }
$$


for any constants $a$ and $b$.

2) If $F \in \mathcal{L}(0, T), \mathbb{E}\left[\sup _{0 \leq s \leq T} F_{s}\right]^{2}<\infty$ and $\sup _{0 \leq s \leq T} \mathbb{E}\left|D_{s}^{\phi} F_{s}\right|^{2}<\infty$, then $\left(\int_{0}^{t} F_{s} d B_{s}^{H}, 0 \leq t \leq T\right)$ has a continuous version.

Property 1) is obvious. To show 2) let $Y_{t}=\int_{0}^{t} F_{s} d B_{s}^{H}, 0 \leq t \leq T$. By the equality (3.17), it follows that

$$
\begin{aligned}
\mathbb{E}\left|Y_{t}-Y_{s}\right|^{2} & =\mathbb{E}\left|\int_{s}^{t} F_{u} d B_{u}^{H}\right|^{2} \\
& \leq \mathbb{E}\left\{\left(\int_{s}^{t} D_{u}^{\phi} F_{u} d u\right)^{2}+\int_{s}^{t} \int_{s}^{t} F_{u} F_{v} \phi(u, v) d u d v\right\} \\
& \leq(t-s) \int_{s}^{t} \mathbb{E}\left|D_{u}^{\phi} F_{u}\right|^{2} d u+\mathbb{E}\left[\sup _{0 \leq s \leq T} F_{s}\right]^{2} \int_{s}^{t} \int_{s}^{t} \phi(u, v) d u d v \\
& \leq(t-s)^{2}+C(t-s)^{2 H} .
\end{aligned}
$$

By the Kolmogorov lemma [22], the property 2) is satisfied.

In the Theorem 3.7, it is not assumed that the stochastic process $\left(F_{s}, s \in[0, T]\right)$ is adapted to the fractional Brownian motion. Now assume that $D_{s}^{\phi} F_{s}=0$ for $s \in[0, T]$. Thus in this case,

$$
\mathbb{E}\left|\int_{0}^{T} F_{s} d B_{s}^{H}\right|^{2}=\mathbb{E}\left\{\int_{0}^{T} \int_{0}^{T} F_{u} F_{v} \phi(u, v) d u d v\right\} .
$$

This fact is stated in the following theorem.

Theorem 3.8 If $F \in \mathcal{L}(0, T)$ and if $F$ satisfies $\mathbb{E} \int_{0}^{T}\left|D_{s}^{\phi} F_{s}\right| d s=0$, then

$$
\mathbb{E}\left|\int_{0}^{T} F_{s} d B_{s}^{H}\right|^{2}=\mathbb{E}\left\{\left|1_{[0, T]} F\right|_{\phi}^{2}\right\}
$$

An analogue of the stochastic integral of Stratonovich type $\int_{0}^{t} F_{s} \delta B_{s}^{H}$ is also introduced. This type of integral is related to the integrals introduced by Lin [17] and Dai and Heyde $[2]$.

Definition 3.3 Let $\left(\pi_{n}, n \in \mathbb{N}\right)$ be a sequence of partitions of $[0, t]$ such that $\left|\pi_{n}\right| \rightarrow 0$ as $n \rightarrow \infty$. If $\sum_{i=0}^{n-1} f\left(t_{i}^{(n)}\right)\left(B^{H}\left(t_{i+1}^{(n)}\right)-B^{H}\left(t_{i}^{(n)}\right)\right)$ converges in $L^{2}(\Omega, \mathcal{F}, P)$ to the same limit for all such sequences $\left(\pi_{n}, n \in \mathbb{N}\right)$, then this limit is called the stochastic integral of Stratonovich type and the limit is denoted by $\int_{0}^{t} f(s) \delta B^{H}(s)$.

Theorem 3.9 If $F \in \mathcal{L}(0, T)$, then the stochastic integral of Stratonovich type $\int_{0}^{t} F_{s} \delta B_{s}^{H}$ exists and the following equality is satisfied

$$
\int_{0}^{t} F_{s} \delta B_{s}^{H}=\int_{0}^{t} F_{s} d B_{s}^{H}+\int_{0}^{t} D_{s}^{\phi} F_{s} d s \quad \text { a.s. }
$$


Proof By Proposition 3.4,

$$
\begin{aligned}
\sum_{i=0}^{n-1} F_{t_{i}^{(n)}}\left(B^{H}\left(t_{i+1}^{(n)}\right)-B^{H}\left(t_{i}^{(n)}\right)\right) & =\sum_{i=0}^{n-1} F_{t_{i}^{(n)}} \diamond\left(B^{H}\left(t_{i+1}^{(n)}\right)-B^{H}\left(t_{i}^{(n)}\right)\right)+\sum_{i=0}^{n-1} D_{\Phi \chi_{\left[t_{i}^{(n)}, t_{i+1}^{(n)}\right]} F_{t_{i}^{(n)}}} \\
& =\sum_{i=0}^{n-1}\left[F_{t_{i}^{(n)}} \diamond\left(B^{H}\left(t_{i+1}^{(n)}\right)-B^{H}\left(t_{i}^{(n)}\right)\right)+\int_{t_{i}^{(n)}}^{t_{i+1}^{(n)}} D_{s}^{\phi} F_{t_{i}^{(n)}} d s\right]
\end{aligned}
$$

This equality easily proves the Theorem.

These two types of stochastic integrals are both interesting.

1) The expectation of $\int_{0}^{t} F_{s} d B_{s}^{H}$ is 0 but the chain rule for this type of integral is more complicated than for the integral of Stratonovich type.

2) The chain rule for the integral of Stratonovich type is simple but $\mathbb{E} \int_{0}^{t} F_{s} \delta B_{s}^{H} \neq 0$ in general.

An example is provided that shows that $\mathbb{E}\left\{\int_{0}^{t} F_{s} \delta B_{s}^{H}\right\}$ is not 0 .

It is well known that if $X$ is a standard normal random variable, $X \sim N(0,1)$, then

$$
\mathbb{E} X^{n}= \begin{cases}\frac{n !}{(\sqrt{2})^{n}(n / 2) !} & \text { if } n \text { is even } \\ 0 & \text { if } n \text { is odd }\end{cases}
$$

Let $f(x)=x^{n}$. If $n$ is odd, then

$$
\begin{aligned}
\mathbb{E} \int_{0}^{t} f\left(B_{s}^{H}\right) \delta B_{s}^{H} & =\mathbb{E} \int_{0}^{t} D_{s}^{\phi} f\left(B_{s}^{H}\right) d s \\
& =\mathbb{E} \int_{0}^{t} f^{\prime}\left(B_{s}^{H}\right) D_{s}^{\phi} B_{s}^{H} d s \\
& =\mathbb{E} \int_{0}^{t} f^{\prime}\left(B_{s}^{H}\right) \int_{0}^{s} \phi(u, s) d u d s \\
& =H \int_{0}^{t} s^{2 H-1} \mathbb{E} f^{\prime}\left(B_{s}^{H}\right) d s \\
& =n H \int_{0}^{t} s^{2 H-1} \mathbb{E}\left(\left(B_{s}^{H}\right)^{n-1}\right) d s \\
& =n H \int_{0}^{t} s^{2 H-1} \mathbb{E}\left(\frac{B_{s}^{H}}{s^{H}}\right)^{n-1} s^{n H-H} d s \\
& =\frac{n ! H t^{(n+1) H}}{\sqrt{2}^{n-1}(n+1) H\left(\frac{n-1}{2}\right) !}
\end{aligned}
$$

which is not 0 . If $n$ is even, then by the same computation,

$$
\mathbb{E} \int_{0}^{t}\left(B_{s}^{H}\right)^{n} \delta B_{s}^{H}=0
$$

Now another interesting phenomenon is shown. Let $\pi$ be a partition of the interval $[0, T]$ : $0=t_{0}<t_{1}<t_{2}<\cdots<t_{n}=T$. Let $(f(s), s \geq 0)$ be a stochastic process on the probability 
space $(\Omega, \mathcal{F}, P)$. For the Brownian motion $\left(B_{t}, t \geq 0\right)$, the Itô integral can be defined as the limit of the Riemann sums $\sum_{i=0}^{n-1} f_{t_{i}}\left(B_{t_{i+1}}-B_{t_{i}}\right)$ as the partition $|\pi| \rightarrow 0$ for smooth $f$. The Stratonovich integral is defined as the limit of the Riemann sums $\sum_{i=0}^{n-1} \frac{f_{t_{i}}+f_{t_{i+1}}}{2}\left(B_{t_{i+1}}-B_{t_{i}}\right)$ as the partition $|\pi| \rightarrow 0$. It may seem to be more natural to define the Stratonovich integral for fractional Brownian motion $\left(B_{t}^{H}, t \geq 0\right)$ in a similar way. It is shown that the above two limits are the same for a large class of stochastic processes.

Initially the following lemma is given.

Lemma 3.10 Let $p$ be a positive even integer. Then

$$
\mathbb{E}\left(B_{t}^{H}-B_{s}^{H}\right)^{p}=\frac{p !}{2^{p / 2}(p / 2) !}|t-s|^{p H} .
$$

Proof By (1.1) it follows that

$$
\begin{aligned}
\mathbb{E}\left|B_{t}^{H}-B_{s}^{H}\right|^{2} & =\mathbb{E}\left(B_{t}^{H}\right)^{2}+\mathbb{E}\left(B_{s}^{H}\right)^{2}-2 \mathbb{E} B_{t}^{H} B_{s}^{H} \\
& =t^{2 H}+s^{2 H}-\left(t^{2 H}+s^{2 H}-|t-s|^{2 H}\right)=|t-s|^{2 H}
\end{aligned}
$$

Thus $\frac{B_{t}^{H}-B_{s}^{H}}{|t-s|^{H}}$ is a standard Gaussian random variable and

$$
\begin{aligned}
\mathbb{E}\left|B_{t}^{H}-B_{s}^{H}\right|^{p} & =|t-s|^{p H} \mathbb{E}\left(\frac{B_{t}^{H}-B_{s}^{H}}{|t-s|^{H}}\right)^{p} \\
& =\frac{p !}{2^{p / 2}(p / 2) !}|t-s|^{p H}
\end{aligned}
$$

Corollary 3.11 For each $\alpha>1$, there is a $C_{\alpha}<\infty$ such that

$$
\mathbb{E}\left|B_{t}^{H}-B_{s}^{H}\right|^{\alpha} \leq C_{\alpha}|t-s|^{\alpha H} .
$$

Definition 3.4 The process $\left(f_{s}, 0 \leq s \leq T\right)$ is said to be a bounded quadratic variation process if there are constants $p \geq 1$ and $0<C_{p}<\infty$ such that for any partition $\pi: 0=t_{0}<$ $t_{1}<t_{2}<\cdots<t_{n}=T$,

$$
\sum_{i=0}^{n-1}\left(\mathbb{E}\left|f_{t_{i+1}}-f_{t_{i}}\right|^{2 p}\right)^{1 / p} \leq C_{p} .
$$

Example: Let $f: \mathbb{R} \rightarrow \mathbb{R}$ be continuously differentiable with bounded first derivative. Then $f\left(B_{s}^{H}\right)$ is a bounded quadratic variation process. In fact, for any $p \geq 1$ and partition $\pi$,

$$
\begin{aligned}
& \sum_{i=0}^{n-1}\left\{\mathbb{E}\left|f\left(B_{t_{i+1}}^{H}\right)-f\left(B_{t_{i}}^{H}\right)\right|^{2 p}\right\}^{1 / p} \\
= & \sum_{i=0}^{n-1}\left\{\mathbb{E}\left(\int_{0}^{1} f^{\prime}\left(B_{t_{i}}^{H}+\theta\left(B_{t_{i+1}}^{H}-B_{t_{i}}^{H}\right)\right) d \theta\left(B_{t_{i+1}}^{H}-B_{t_{i}}^{H}\right)\right)^{2 p}\right\}^{1 / p} \\
\leq & C \sum_{i=0}^{n-1} \mathbb{E}\left(\left|B_{t_{i+1}}^{H}-B_{t_{i}}^{H}\right|^{2 p}\right)^{1 / p} \\
\leq & C \sum_{i=0}^{n-1}\left|t_{i+1}-t_{i}\right|^{2 H} \leq C T
\end{aligned}
$$


Theorem 3.12 Let $(f(t), 0 \leq t \leq T)$ be a bounded quadratic variation process. Let $\left(\pi_{n}, n \in\right.$ $\mathbb{N})$ be a sequence of partitions of $[0, T]$ such that $\left|\pi_{n}\right| \rightarrow 0$ as $n \rightarrow \infty$ and

$$
\left(\sum_{i=0}^{n-1} f\left(t_{i}^{(n)}\right)\left(B^{H}\left(t_{i+1}^{(n)}\right)-B^{H}\left(t_{i}^{(n)}\right)\right), n \in \mathbb{N}\right)
$$

converges to a random variable $G$ in $L^{2}(\Omega, \mathcal{F}, P)$, where $\pi_{n}=\left\{t_{0}^{(n)}, \cdots, t_{n}^{(n)}\right\}$. Then

$$
\left(\sum_{i=0}^{n-1} f\left(t_{i+1}^{(n)}\right)\left(B^{H}\left(t_{i+1}^{(n)}\right)-B^{H}\left(t_{i}^{(n)}\right)\right), n \in \mathbb{N}\right)
$$

also converges to $G$ in $L^{2}(\Omega, \mathcal{F}, P)$.

Proof It suffices to show that $\sum_{i=0}^{n-1}\left(f_{t_{i+1}}-f_{t_{i}}\right)\left(B_{t_{i+1}}^{H}-B_{t_{i}}^{H}\right)$ converges to 0 in $L^{2}(\Omega, \mathcal{F}, P)$. Let $p$ be a number as indicated in the definition of bounded quadratic variation for $\left(f_{t}, 0 \leq t \leq T\right)$

$$
\begin{aligned}
& \left(\mathbb{E}\left\{\sum_{i=0}^{n-1}\left(f_{t_{i+1}}-f_{t_{i}}\right)\left(B_{t_{i+1}}^{H}-B_{t_{i}}^{H}\right)\right\}^{2}\right)^{1 / 2} \\
\leq & \sum_{i=0}^{n-1}\left(\mathbb{E}\left(f_{t_{i+1}}-f_{t_{i}}\right)^{2} \mathbb{E}\left(B_{t_{i+1}}^{H}-B_{t_{i}}^{H}\right)^{2}\right)^{1 / 2} \\
\leq & \sum_{i=0}^{n-1}\left(\mathbb{E}\left(f_{t_{i+1}}-f_{t_{i}}\right)^{2 p}\right)^{1 / 2 p}\left(\mathbb{E}\left(B_{t_{i+1}}^{H}-B_{t_{i}}^{H}\right)^{2 q}\right)^{1 / 2 q} \\
\leq & \left\{\sum_{i=0}^{n-1}\left(\mathbb{E}\left(f_{t_{i+1}}-f_{t_{i}}\right)^{2 p}\right)^{1 / p}\right\}^{1 / 2}\left\{\sum_{i=0}^{n-1}\left(\mathbb{E}\left|B_{t_{i+1}}^{H}-B_{t_{i}}^{H}\right|^{2 q}\right)^{1 / q}\right\}^{1 / 2} \\
\leq & C\left\{\sum_{i=0}^{n-1}\left|t_{i+1}-t_{i}\right|^{2 H}\right\}^{1 / 2} \\
\leq & C \max _{0 \leq i \leq n-1}\left(t_{i+1}-t_{i}\right)^{H-\frac{1}{2}}\left\{\sum_{i=0}^{n-1}\left|t_{i+1}-t_{i}\right|\right\}^{\frac{1}{2}} \\
\leq & C \sqrt{T} \max _{0 \leq i \leq n-1}\left(t_{i+1}-t_{i}\right)^{H-\frac{1}{2}} \rightarrow 0 \quad(\text { as }|\pi| \rightarrow 0),
\end{aligned}
$$

where $1 / p+1 / q=1$.

It can also be shown with a slightly more lengthy argument that if $\left(f_{s}, s \geq 0\right)$ is a process with bounded quadratic variation and $\xi_{i}$ is any point in $\left[t_{i}, t_{i+1}\right]$, then the limit of the Riemann sums $\sum_{i=0}^{n-1} f_{\xi_{i}}\left(B_{t_{i+1}}^{H}-B_{t_{i}}^{H}\right)$ converge in $L^{2}(\Omega, \mathcal{F}, P)$ to $\int_{0}^{T} f_{s} \delta B_{s}^{H}$ if it is true for any particular choice of such a $\xi_{i}$.

\section{An Itô formula}

Now an analogue of the Itô formula is established, that is, a chain rule for the integral introduced in the last section. Let $f: \mathbb{R} \rightarrow \mathbb{R}$ be a twice continuously differentiable function 
with bounded second derivative. Then for a partition $\left\{t_{0}, t_{1}, \cdots, t_{n}\right\}$ of $[0, T]$ it follows by Taylor's formula that

$$
\begin{aligned}
f\left(B_{T}^{H}\right)-f(0)= & \sum_{i=0}^{n-1}\left[f\left(B_{t_{i+1}}^{H}\right)-f\left(B_{t_{i}}^{H}\right)\right] \\
= & \sum_{i=0}^{n-1} f^{\prime}\left(B_{t_{i}}^{H}\right)\left[B_{t_{i+1}}^{H}-B_{t_{i}}^{H}\right]+\frac{1}{2} \sum_{i=0}^{n-1} f^{\prime \prime}\left(\xi_{i}\right)\left[B_{t_{i+1}}^{H}-B_{t_{i}}^{H}\right]^{2} \\
= & \sum_{i=0}^{n-1} f^{\prime}\left(B_{t_{i}}^{H}\right) \diamond\left[B_{t_{i+1}}^{H}-B_{t_{i}}^{H}\right]+\sum_{i=0}^{n-1} \int_{t_{i}}^{t_{i+1}} D_{s}^{\phi} f^{\prime}\left(B_{t_{i}}^{H}\right) d s \\
& \quad+\frac{1}{2} \sum_{i=0}^{n-1} f^{\prime \prime}\left(\xi_{i}\right)\left[B_{t_{i+1}}^{H}-B_{t_{i}}^{H}\right]^{2} \\
= & I_{1}+I_{2}+I_{3},
\end{aligned}
$$

where $\xi_{i} \in\left(B_{t_{i}}^{H}, B_{t_{i+1}}^{H}\right)$. Since it is assumed that $H>1 / 2$, it follows that $I_{3} \rightarrow 0$ in $L^{2}(\Omega, \mathcal{F}, P)$. By the definition of the stochastic integral introduced in the preceding section, the first term $I_{1}$ converges to $\int_{0}^{T} f^{\prime}\left(B_{s}^{H}\right) d B_{s}^{H}$ in $L^{2}$. By a version of the chain rule for the $\phi$-differentiation operator, it follows that for $s \in\left[t_{i}, t_{i+1}\right)$

$$
\begin{aligned}
D_{s}^{\phi} f^{\prime}\left(B_{t_{i}}^{H}\right) & =f^{\prime \prime}\left(B_{t_{i}}^{H}\right) D_{s}^{\phi} B_{t_{i}}^{H} \\
& =f^{\prime \prime}\left(B_{t_{i}}^{H}\right) \int_{0}^{t_{i}} \phi(u, s) d u \\
& =H f^{\prime \prime}\left(B_{t_{i}}^{H}\right)\left[s^{2 H-1}-\left(s-t_{i}\right)^{2 H-1}\right]
\end{aligned}
$$

Thus the second sum in the three sums from Taylor's formula converges to $H \int_{0}^{T} s^{2 H-1} f^{\prime \prime}\left(B_{s}^{H}\right) d s$ in $L^{2}$. The following chain rule formula is obtained.

Theorem 4.1 If $f: \mathbb{R} \rightarrow \mathbb{R}$ is a twice continuously differentiable function with bounded derivatives to order two, then

$$
f\left(B_{T}^{H}\right)-f\left(B_{0}^{H}\right)=\int_{0}^{T} f^{\prime}\left(B_{s}^{H}\right) d B_{s}^{H}+H \int_{0}^{T} s^{2 H-1} f^{\prime \prime}\left(B_{s}^{H}\right) d s \quad \text { a.s. }
$$

It is interesting to note that this formula implies the usual Itô formula for Brownian motion when $H=1 / 2$ is formally substituted in (4.1).

The following theorem shows how to compute the $\phi$-derivative of a stochastic integral of Itô type. It can be verified from the product rule and the Riemann sum approximations to the stochastic integral.

Theorem 4.2 Let $\left(F_{t}, t \in[0, T]\right)$ be a stochastic process in $\mathcal{L}(0, T)$ and $\sup _{0 \leq s \leq T} \mathbb{E}\left|D_{s}^{\phi} F_{s}\right|^{2}<$ $\infty$ and let $\eta_{t}=\int_{0}^{t} F_{u} d B_{u}^{H}$ for $t \in[0, T]$. Then for $s, t \in[0, T]$

$$
D_{s}^{\phi} \eta_{t}=\int_{0}^{t} D_{s}^{\phi} F_{u} d B_{u}^{H}+\int_{0}^{t} F_{u} \phi(s, u) d u, \quad \text { a.s. }
$$


Now a more general Itô formula is given.

Theorem 4.3 Let $\eta_{t}=\int_{0}^{t} F_{u} d B_{u}^{H}$, where $\left(F_{u}, 0 \leq u \leq T\right)$ is a stochastic process in $\mathcal{L}(0, T)$. Assume that there is an $\alpha>1-H$ such that

$$
\mathbb{E}\left|F_{u}-F_{v}\right|^{2} \leq C|u-v|^{2 \alpha} .
$$

where $|u-v| \leq \delta$ for some $\delta>0$ and

$$
\lim _{0 \leq u, v \leq t,|u-v| \rightarrow 0} \mathbb{E}\left|D_{u}^{\phi}\left(F_{u}-F_{v}\right)\right|^{2}=0 .
$$

Let $f: \mathbb{R}_{+} \times \mathbb{R} \rightarrow \mathbb{R}$ be a function having the first continuous derivative in its first variable and the second continuous derivative in its second variable. Assume that these derivatives are bounded. Moreover, it is assumed that $\mathbb{E} \int_{0}^{T}\left|F_{s} D_{s}^{\phi} \eta_{s}\right| d s<\infty$ and $\left(f^{\prime}\left(s, \eta_{s}\right) F_{s}, s \in[0, T]\right)$ is in $\mathcal{L}(0, T)$. Then for $0 \leq t \leq T$,

$$
\begin{gathered}
f\left(t, \eta_{t}\right)=f(0,0)+\int_{0}^{t} \frac{\partial f}{\partial s}\left(s, \eta_{s}\right) d s+\int_{0}^{t} \frac{\partial f}{\partial x}\left(s, \eta_{s}\right) F_{s} d B_{s}^{H} \\
+\int_{0}^{t} \frac{\partial^{2} f}{\partial x^{2}}\left(s, \eta_{s}\right) F_{s} D_{s}^{\phi} \eta_{s} d s . \quad \text { a.s. }
\end{gathered}
$$

Proof Let $\pi$ be a partition defined as above by replacing $T$ by $t$. Then

$$
\begin{aligned}
f\left(t, \eta_{t}\right)-f(0,0) & =\sum_{k=0}^{n-1}\left[f\left(t_{k+1}, \eta_{t_{k+1}}\right)-f\left(t_{k}, \eta_{t_{k}}\right)\right] \\
& =\sum_{k=0}^{n-1}\left[f\left(t_{k+1}, \eta_{t_{k+1}}\right)-f\left(t_{k}, \eta_{t_{k+1}}\right)\right]+\sum_{k=0}^{n-1}\left[f\left(t_{k}, \eta_{t_{k+1}}\right)-f\left(t_{k}, \eta_{t_{k}}\right)\right]
\end{aligned}
$$

by the mean value theorem. It is easy to see that the first sum converges to $\int_{0}^{t} \frac{\partial f}{\partial s}\left(s, \eta_{s}\right) d s$ in $L^{2}$. Now consider the second sum. Using Taylor's formula, it follows that

$$
f\left(t_{k}, \eta_{t_{k+1}}\right)-f\left(t_{k}, \eta_{t_{k}}\right)=\frac{\partial f}{\partial x}\left(t_{k}, \eta_{t_{k}}\right)\left(\eta_{t_{k+1}}-\eta_{t_{k}}\right)+\frac{1}{2} \frac{\partial^{2} f}{\partial x^{2}}\left(t_{k}, \tilde{\eta}_{t_{k}}\right)\left(\eta_{t_{k+1}}-\eta_{t_{k}}\right)^{2}
$$

where $\tilde{\eta}_{t_{k}} \in\left(\eta_{t_{k}}, \eta_{t_{k+1}}\right)$. An upper bound is obtained for $\mathbb{E}\left(\eta_{t_{k+1}}-\eta_{t_{k}}\right)^{2}$ as follows

$$
\begin{aligned}
\mathbb{E}\left(\eta_{t_{k+1}}-\eta_{t_{k}}\right)^{2} & =\mathbb{E}\left(\int_{t_{k}}^{t_{k+1}} D_{s}^{\phi} F_{s} d s\right)^{2}+\mathbb{E} \int_{t_{k}}^{t_{k+1}} \int_{t_{k}}^{t_{k+1}} F_{u} F_{v} \phi(u, v) d u d v \\
& \leq\left(t_{k+1}-t_{k}\right) \int_{t_{k}}^{t_{k+1}} \mathbb{E}\left(D_{s}^{\phi} F_{s}\right)^{2} d s+\int_{t_{k}}^{t_{k+1}} \int_{t_{k}}^{t_{k+1}}\left(\mathbb{E} F_{u}^{2}\right)^{1 / 2}\left(\mathbb{E} F_{v}^{2}\right)^{1 / 2} \phi(u, v) d u d v \\
& \leq C\left[\left(t_{k+1}-t_{k}\right)^{2}+\int_{t_{k}}^{t_{k+1}} \int_{t_{k}}^{t_{k+1}} \phi(u, v) d u d v\right] \\
& \leq C\left(t_{k+1}-t_{k}\right)^{2}+C\left(t_{k+1}-t_{k}\right)^{2 H} \leq C\left(t_{k+1}-t_{k}\right)^{2 H}
\end{aligned}
$$


where $t_{i+1}-t_{i}<1$ and $C$ is a constant independent of the partition $\pi$, that may differ for different applications.

Thus

$$
\begin{aligned}
\mathbb{E} \sum_{k=0}^{n-1} \frac{\partial^{2} f}{\partial x^{2}}\left(t_{k}, \tilde{\eta}_{t_{k}}\right)\left(\eta_{t_{k+1}}-\eta_{t_{k}}\right)^{2} & \leq C \sum_{k=0}^{n-1} \mathbb{E}\left(\eta_{t_{k+1}}-\eta_{t_{k}}\right)^{2} \\
& \leq C \sum_{k=0}^{n-1}\left(t_{k+1}-t_{k}\right)^{2 H} \\
& \rightarrow 0 \quad \text { as }|\pi| \rightarrow 0
\end{aligned}
$$

On the other hand

$$
\begin{aligned}
\frac{\partial f}{\partial x}\left(t_{k}, \eta_{t_{k}}\right)\left(\eta_{t_{k+1}}-\eta_{t_{k}}\right)= & \frac{\partial f}{\partial x}\left(t_{k}, \eta_{t_{k}}\right)\left(F_{t_{k}} \diamond\left(B_{t_{k+1}}^{H}-B_{t_{k}}^{H}\right)\right) \\
& +\frac{\partial f}{\partial x}\left(t_{k}, \eta_{t_{k}}\right)\left(\int_{t_{k}}^{t_{k+1}}\left(F_{s}-F_{t_{k}}\right) d B_{s}^{H}\right)
\end{aligned}
$$

The first term on the right hand side can be expressed as

$$
\begin{aligned}
& \frac{\partial f}{\partial x}\left(t_{k}, \eta_{t_{k}}\right)\left(F_{t_{k}} \diamond\left(B_{t_{k+1}}^{H}-B_{t_{k}}^{H}\right)\right) \\
= & \frac{\partial f}{\partial x}\left(t_{k}, \eta_{t_{k}}\right)\left(F_{t_{k}}\left(B_{t_{k+1}}^{H}-B_{t_{k}}^{H}\right)-\int_{t_{k}}^{t_{k+1}} D_{s}^{\phi} F_{t_{k}} d s\right) \\
= & \frac{\partial f}{\partial x}\left(t_{k}, \eta_{t_{k}}\right) F_{t_{k}}\left(B_{t_{k+1}}^{H}-B_{t_{k}}^{H}\right)-\frac{\partial f}{\partial x}\left(t_{k}, \eta_{t_{k}}\right) \int_{t_{k}}^{t_{k+1}} D_{s}^{\phi} F_{t_{k}} d s \\
= & {\left[\frac{\partial f}{\partial x}\left(t_{k}, \eta_{t_{k}}\right) F_{t_{k}}\right] \diamond\left(B_{t_{k+1}}^{H}-B_{t_{k}}^{H}\right)+\int_{t_{k}}^{t_{k+1}} D_{s}^{\phi}\left(\frac{\partial f}{\partial x}\left(t_{k}, \eta_{t_{k}}\right) F_{t_{k}}\right) d s } \\
& -\frac{\partial f}{\partial x}\left(t_{k}, \eta_{t_{k}}\right) \int_{t_{k}}^{t_{k+1}} D_{s}^{\phi} F_{t_{k}} d s \\
= & {\left[\frac{\partial f}{\partial x}\left(t_{k}, \eta_{t_{k}}\right) F_{t_{k}}\right] \diamond\left(B_{t_{k+1}}^{H}-B_{t_{k}}^{H}\right)+\int_{t_{k}}^{t_{k+1}} F_{t_{k}} D_{s}^{\phi} \frac{\partial f}{\partial x}\left(t_{k}, \eta_{t_{k}}\right) d s }
\end{aligned}
$$

Thus

$$
\begin{aligned}
\sum_{k=0}^{n-1} \frac{\partial f}{\partial x}\left(t_{k}, \eta_{t_{k}}\right)\left(\eta_{t_{k+1}}-\eta_{t_{k}}\right)= & \sum_{k=0}^{n-1}\left[\frac{\partial f}{\partial x}\left(t_{k}, \eta_{t_{k}}\right) F_{t_{k}}\right] \diamond\left(B_{t_{k+1}}^{H}-B_{t_{k}}^{H}\right) \\
& +\sum_{k=0}^{n-1} \int_{t_{k}}^{t_{k+1}} F_{t_{k}} D_{s}^{\phi} \frac{\partial f}{\partial x}\left(t_{k}, \eta_{t_{k}}\right) d s
\end{aligned}
$$

As $|\pi| \rightarrow 0$, the first term converges to

$$
\int_{0}^{t} F_{s} \frac{\partial f}{\partial x}\left(s, \eta_{s}\right) d B_{s}^{H}
$$


in $L^{2}$ and the second term converges to

$$
\int_{0}^{t} \frac{\partial^{2} f}{\partial x^{2}}\left(s, \eta_{s}\right) D_{s}^{\phi} \eta_{s} F_{s} d s
$$

in $L^{2}$. To prove the theorem it is only necessary to show that as $|\pi| \rightarrow 0$,

$$
\sum_{k=0}^{n-1} \mathbb{E}\left|\frac{\partial f}{\partial x}\left(t_{k}, \eta_{t_{k}}\right) \int_{t_{k}}^{t_{k+1}}\left(F_{s}-F_{t_{k}}\right) d B_{s}^{H}\right| \rightarrow 0
$$

Since $f$ has a bounded second derivative, it follows that

$$
\left|\frac{\partial f}{\partial x}\left(t_{k}, \eta_{t_{k}}\right)\right| \leq C\left(1+\left|\eta_{t_{k}}\right|\right)
$$

Thus

$$
\mathbb{E}\left|\frac{\partial f}{\partial x}\left(t_{k}, \eta_{t_{k}}\right)\right|^{2} \leq C
$$

Furthermore

$$
\begin{aligned}
& \sum_{k=0}^{n-1} \mathbb{E}\left|\frac{\partial f}{\partial x}\left(t_{k}, \eta_{t_{k}}\right) \int_{t_{k}}^{t_{k+1}}\left(F_{s}-F_{t_{k}}\right) d B_{s}^{H}\right| \\
\leq & C \sum_{k=0}^{n-1}\left\{\mathbb{E}\left|\int_{t_{k}}^{t_{k+1}}\left(F_{s}-F_{t_{k}}\right) d B_{s}^{H}\right|^{2}\right\}^{1 / 2} \\
= & C \sum_{k=0}^{n-1}\left\{\mathbb{E}\left(\int_{t_{k}}^{t_{k+1}}\left(D_{s}^{\phi}\left(F_{s}-F_{t_{k}}\right)\right) d s\right)^{2}+\mathbb{E} \int_{t_{k}}^{t_{k+1}} \int_{t_{k}}^{t_{k+1}}\left(F_{u}-F_{t_{k}}\right)\left(F_{v}-F_{t_{k}}\right) \phi(u, v) d u d v\right\}^{1 / 2} \\
\leq & C \sum_{k=0}^{n-1}\left\{\left(t_{k+1}-t_{k}\right) \int_{t_{k}}^{t_{k+1}} \mathbb{E}\left(D_{s}^{\phi}\left(F_{s}-F_{t_{k}}\right)\right)^{2} d s\right. \\
& \left.+\int_{t_{k}}^{t_{k+1}} \int_{t_{k}}^{t_{k+1}}\left\{\mathbb{E}\left(F_{u}-F_{t_{k}}\right)^{2}\right\}^{1 / 2}\left\{\mathbb{E}\left(F_{v}-F_{t_{k}}\right)^{2}\right\}^{1 / 2} \phi(u, v) d u d v\right\}^{1 / 2} \\
\leq & C \sum_{k=0}^{n-1}\left[\sup _{t_{k} \leq s \leq t_{k+1}} \mathbb{E}\left|D_{s}^{\phi}\left(F_{s}-F_{t_{k}}\right)\right|^{2}\left(t_{k+1}-t_{k}\right)^{2}+\left(t_{k+1}-t_{k}\right)^{2 H}\left\{\sup _{t_{k} \leq s \leq t_{k+1}} \mathbb{E}\left(F_{s}-F_{t_{k}}\right)^{2}\right\}\right]^{1 / 2} \\
\leq & C\left\{\sup _{t_{k} \leq s \leq t_{k+1}} \mathbb{E}\left|D_{s}^{\phi}\left(F_{s}-F_{t_{k}}\right)\right|^{2}\right\}^{1 / 2}+C|\pi|^{H+\alpha-1} \\
\rightarrow & 0
\end{aligned}
$$

as $|\pi| \rightarrow 0$. This proves the theorem.

The equality (4.2) can be formally expressed as

$$
d f\left(t, \eta_{t}\right)=\frac{\partial f}{\partial t}\left(t, \eta_{t}\right) d t+\frac{\partial f}{\partial x}\left(t, \eta_{t}\right) F_{t} d B_{t}^{H}+\frac{\partial^{2} f}{\partial x^{2}}\left(t, \eta_{t}\right) F_{t} D_{t}^{\phi} \eta_{t} d t
$$

If $F(s)=a(s)$ is a deterministic function then (4.1) simplifies as follows. 
Corollary 4.4 Let $\eta_{t}=\int_{0}^{t} a_{u} d B_{u}^{H}$, where $a \in L_{\phi}^{2}$ and $f: \mathbb{R}_{+} \times \mathbb{R} \rightarrow \mathbb{R}$ satisfies the conditions in Theorem 4.3. Let $\left(\frac{\partial f}{\partial x}\left(s, \eta_{s}\right) a_{s}, s \in[0, T]\right)$ be in $\mathcal{L}(0, T)$. Then

$$
\begin{aligned}
f\left(t, \eta_{t}\right)=f(0,0) & +\int_{0}^{t} \frac{\partial f}{\partial s}\left(s, \eta_{s}\right) d s+\int_{0}^{t} \frac{\partial f}{\partial x}\left(s, \eta_{s}\right) a_{s} d B_{s}^{H} \\
& +\int_{0}^{t} \frac{\partial^{2} f}{\partial x^{2}}\left(s, \eta_{s}\right) \int_{0}^{s} \phi(s, v) a_{v} d v d s \quad a . s .
\end{aligned}
$$

or formally

$$
\begin{aligned}
d f\left(t, \eta_{t}\right)=\frac{\partial f}{\partial t}( & \left.t, \eta_{t}\right) d t+\frac{\partial f}{\partial x}\left(t, \eta_{t}\right) a_{t} d B_{t}^{H} \\
& +\frac{\partial^{2} f}{\partial x^{2}}\left(t, \eta_{t}\right) \int_{0}^{t} \phi(t, v) a_{v} d v d t .
\end{aligned}
$$

If $a_{s} \equiv 1$, then Theorem 4.1 is obtained.

In the classical stochastic analysis, the stochastic integral can be defined for general semimartingales and an Itô formula can be given. By the Doob-Meyer decomposition [4], a semimartingale can be expressed as the sum of a martingale and a bounded variation process. A semimartingale $\left(X_{t}, t \geq 0\right)$ with respect to a Brownian motion can often be expressed as $X_{t}=X_{0}+\int_{0}^{t} f_{s} d B_{s}+\int_{0}^{t} g_{s} d s$. An Itô formula in the analogous form with respect to fractional Brownian motions is given. This generalization of the Itô formula is useful in applications.

Theorem 4.5 Let $\left(F_{u}, u \in[0, T]\right)$ satisfy the conditions of Theorem 4.3 and let $\mathbb{E} \sup _{0 \leq s \leq T}\left|G_{s}\right|<$ $\infty$. Denote $\eta_{t}=\xi+\int_{0}^{t} G_{u} d u+\int_{0}^{t} F_{u} d B_{u}^{H}, \xi \in \mathbb{R}$ for $t \in[0, T]$. Let $\left(\frac{\partial f}{\partial x}\left(s, \eta_{s}\right) F_{s}, s \in[0, T]\right) \in$ $\mathcal{L}(0, T)$. Then for $t \in[0, T]$

$$
\begin{aligned}
f\left(t, \eta_{t}\right)= & f(0, \xi)+\int_{0}^{t} \frac{\partial f}{\partial s}\left(s, \eta_{s}\right) d s+\int_{0}^{t} \frac{\partial f}{\partial x}\left(s, \eta_{s}\right) G_{s} d s \\
& +\int_{0}^{t} \frac{\partial f}{\partial x}\left(s, \eta_{s}\right) F_{s} d B_{s}^{H}+\int_{0}^{t} \frac{\partial^{2} f}{\partial x^{2}}\left(s, \eta_{s}\right) F_{s} D_{s}^{\phi} \eta_{s} d s \quad \text { a.s }
\end{aligned}
$$

The proof is the same as for the above theorem.

Now the Itô formula for $\mathbb{R}^{n}$-valued processes is given.

Theorem 4.6 Let $\left(F_{s}^{i}, i=1, \cdots, n, s \in[0, T]\right)$ satisfy the conditions of Theorem 4.3 for $F$. Let $\xi_{t}^{k}=\int_{0}^{t} F_{s}^{k} d B_{s}^{H}, k=1,2, \cdots, n$ for $t \in[0, T]$. For $k=1,2, \cdots n$ let $\left(f_{x_{k}}\left(s, \eta_{s}\right) F_{s}^{k}, s \in\right.$ $[0, T])$ be in $\mathcal{L}(0, T)$. Let $f$ be twice continuously differentiable with bounded derivatives to second order. Then

$$
\begin{aligned}
f\left(t, \xi_{t}^{1}, \cdots, \xi_{t}^{n}\right)= & f(0,0, \cdots, 0)+\int_{0}^{t} f_{s}\left(s, \xi_{s}^{1}, \cdots, \xi_{s}^{n}\right) d s \\
& +\sum_{k=1}^{n} \int_{0}^{t} f_{x_{k}}\left(s, \xi_{s}^{1}, \cdots, \xi_{s}^{n}\right) F_{s}^{k} d B_{s}^{H} \\
& +\sum_{k, l=1}^{n} \int_{0}^{t} f_{x_{k} x_{l}}\left(s, \xi_{s}^{1}, \cdots, \xi_{s}^{n}\right) F_{s}^{k} D_{s}^{\phi} \xi_{s}^{l} d s \quad \text { a.s. }
\end{aligned}
$$


Proof The theorem is verified for $n=2$. To simplify the notation, let $F^{1}=F$ and $F^{2}=G$. Let $\pi: 0=t_{0}<t_{1}<t_{2}<\cdots<t_{n}=t$ be a partition of the interval $[0, t]$. Then

$$
\begin{aligned}
f\left(t, \xi_{t}, \eta_{t}\right)-f(0,0,0)=\sum_{k=0}^{n-1}( & \left.f\left(t_{k+1}, \xi_{t_{k+1}}, \eta_{t_{k+1}}\right)-f\left(t_{k}, \xi_{t_{k+1}}, \eta_{t_{k+1}}\right)\right) \\
& +\sum_{k=0}^{n-1}\left(f\left(t_{k}, \xi_{t_{k+1}}, \eta_{t_{k+1}}\right)-f\left(t_{k}, \xi_{t_{k}}, \eta_{t_{k}}\right)\right)
\end{aligned}
$$

by the mean value theorem. It is easy to see that the first sum converges to $\int_{0}^{t} \frac{\partial f}{\partial t} d s$ in $L^{2}$ as $|\pi| \rightarrow 0$. To determine the limit of the second sum, consider each term in the sum:

$$
\begin{aligned}
& f\left(t_{k}, \xi_{t_{k+1}}, \eta_{t_{k+1}}\right)-f\left(t_{k}, \xi_{t_{k}}, \eta_{t_{k}}\right) \\
= & f_{x}\left(t_{k}, \xi_{t_{k}}, \eta_{t_{k}}\right)\left(\xi_{t_{k+1}}-\xi_{t_{k}}\right)+f_{y}\left(t_{k}, \xi_{t_{k}}, \eta_{t_{k}}\right)\left(\eta_{t_{k+1}}-\eta_{t_{k}}\right) \\
& +\frac{1}{2} f_{x x}\left(t_{k}, \tilde{\xi}_{t_{k}}, \tilde{\eta}_{t_{k}}\right)\left(\xi_{t_{k+1}}-\xi_{t_{k}}\right)^{2}+\frac{1}{2} f_{y y}\left(t_{k}, \tilde{\xi}_{t_{k}}, \tilde{\eta}_{t_{k}}\right)\left(\eta_{t_{k+1}}-\eta_{t_{k}}\right)^{2} \\
& +f_{x y}\left(t_{k}, \tilde{\xi}_{t_{k}}, \tilde{\eta}_{t_{k}}\right)\left(\xi_{t_{k+1}}-\xi_{t_{k}}\right)\left(\eta_{t_{k+1}}-\eta_{t_{k}}\right)
\end{aligned}
$$

where $\tilde{\xi}_{t_{k}} \in\left(\xi_{t_{k}}, \xi_{t_{k+1}}\right)$ and $\tilde{\eta}_{t_{k}} \in\left(\eta_{t_{k}}, \eta_{t_{k+1}}\right)$. Thus

$$
\begin{aligned}
& \sum_{k=0}^{n-1}\left(f\left(t_{k}, \xi_{t_{k+1}}, \eta_{t_{k+1}}\right)-f\left(t_{k}, \xi_{t_{k}}, \eta_{t_{k}}\right)\right) \\
= & \sum_{k=0}^{n-1} f_{x}\left(t_{k}, \xi_{t_{k}}, \eta_{t_{k}}\right)\left(\xi_{t_{k+1}}-\xi_{t_{k}}\right)+\sum_{k=0}^{n-1} f_{y}\left(t_{k}, \xi_{t_{k}}, \eta_{t_{k}}\right)\left(\eta_{t_{k+1}}-\eta_{t_{k}}\right) \\
& +\frac{1}{2} \sum_{k=0}^{n-1} f_{x x}\left(t_{k}, \tilde{\xi}_{t_{k}}, \tilde{\eta}_{t_{k}}\right)\left(\xi_{t_{k+1}}-\xi_{t_{k}}\right)^{2} \\
& +\frac{1}{2} \sum_{k=0}^{n-1} f_{y y}\left(t_{k}, \tilde{\xi}_{t_{k}}, \tilde{\eta}_{t_{k}}\right)\left(\eta_{t_{k+1}}-\eta_{t_{k}}\right)^{2} \\
& +\sum_{k=0}^{n-1} f_{x y}\left(t_{k}, \tilde{\xi}_{t_{k}}, \tilde{\eta}_{t_{k}}\right)\left(\xi_{t_{k+1}}-\xi_{t_{k}}\right)\left(\eta_{t_{k+1}}-\eta_{t_{k}}\right) \\
= & I_{1}^{\pi}+I_{2}^{\pi}+I_{3}^{\pi}+I_{4}^{\pi}+I_{5}^{\pi}
\end{aligned}
$$

In a similar way as the proof of Theorem 4.3 , it can be shown that as $|\pi| \rightarrow 0, I_{k}^{\pi}$ converges to 0 in $L^{2}$ for $k=3,4,5$.

$f_{x}\left(t_{k}, \xi_{t_{k}}, \eta_{t_{k}}\right)\left(\xi_{t_{k+1}}-\xi_{t_{k}}\right)=f_{x}\left(t_{k}, \xi_{t_{k}}, \eta_{t_{k}}\right)\left(F_{t_{k}} \diamond\left(B_{t_{k+1}}^{H}-B_{t_{k}}^{H}\right)\right)+f_{x}\left(t_{k}, \xi_{t_{k}}, \eta_{t_{k}}\right)\left(\int_{t_{k}}^{t_{k+1}}\left(F_{s}-F_{t_{k}}\right) d B_{s}^{H}\right)$

In a similar way to the proof of Theorem 4.3 the second sum does not contribute as $|\pi| \rightarrow 0$.

By the definition of the Wick product it follows that

$$
f_{x}\left(t_{k}, \xi_{t_{k}}, \eta_{t_{k}}\right)\left(F_{t_{k}} \diamond\left(B_{t_{k+1}}^{H}-B_{t_{k}}^{H}\right)\right)
$$




$$
\begin{aligned}
&= f_{x}\left(t_{k}, \xi_{t_{k}}, \eta_{t_{k}}\right)\left(F_{t_{k}}\left(B_{t_{k+1}}^{H}-B_{t_{k}}^{H}\right)-\int_{t_{k}}^{t_{k+1}} D_{s}^{\phi} F_{t_{k}} d s\right) \\
&= f_{x}\left(t_{k}, \xi_{t_{k}}, \eta_{t_{k}}\right) F_{t_{k}}\left(B_{t_{k+1}}^{H}-B_{t_{k}}^{H}\right)-f_{x}\left(t_{k}, \xi_{t_{k}}, \eta_{t_{k}}\right) \int_{t_{k}}^{t_{k+1}} D_{s}^{\phi} F_{t_{k}} d s \\
&=\left(f_{x}\left(t_{k}, \xi_{t_{k}}, \eta_{t_{k}}\right) F_{t_{k}}\right) \diamond\left(B_{t_{k+1}}^{H}-B_{t_{k}}^{H}\right)+\int_{t_{k}}^{t_{k+1}} D_{s}^{\phi}\left(f_{x}\left(t_{k}, \xi_{t_{k}}, \eta_{t_{k}}\right) F_{t_{k}}\right) d s \\
& \quad f_{x}\left(t_{k}, \xi_{t_{k}}, \eta_{t_{k}}\right) \int_{t_{k}}^{t_{k+1}} D_{s}^{\phi} F_{t_{k}} d s \\
&=\left(f_{x}\left(t_{k}, \xi_{t_{k}}, \eta_{t_{k}}\right) F_{t_{k}}\right) \diamond\left(B_{t_{k+1}}^{H}-B_{t_{k}}^{H}\right)+\int_{t_{k}}^{t_{k+1}} f_{x x}\left(t_{k}, \xi_{t_{k}}, \eta_{t_{k}}\right) D_{s}^{\phi} \xi_{t_{k}} F_{t_{k}} d s \\
&+\int_{t_{k}}^{t_{k+1}} f_{x y}\left(t_{k}, \xi_{t_{k}}, \eta_{t_{k}}\right) D_{s}^{\phi} \eta_{t_{k}} F_{t_{k}} d s+\int_{t_{k}}^{t_{k+1}} f_{x}\left(t_{k}, \xi_{t_{k}}, \eta_{t_{k}}\right) D_{s}^{\phi} F_{t_{k}} d s \\
& \quad-f_{x}\left(t_{k}, \xi_{t_{k}}, \eta_{t_{k}}\right) \int_{t_{k}}^{t_{k+1}} D_{s}^{\phi} F_{t_{k}} d s \\
&=\left(f_{x}\left(t_{k}, \xi_{t_{k}}, \eta_{t_{k}}\right) F_{t_{k}}\right) \diamond\left(B_{t_{k+1}}^{H}-B_{t_{k}}^{H}\right)+\int_{t_{k}}^{t_{k+1}} f_{x x}\left(t_{k}, \xi_{t_{k}}, \eta_{t_{k}}\right) D_{s}^{\phi} \xi_{t_{k}} F_{t_{k}} d s \\
& \quad+\int_{t_{k}}^{t_{k+1}} f_{x y}\left(t_{k}, \xi_{t_{k}}, \eta_{t_{k}}\right) D_{s}^{\phi} \eta_{t_{k}} F_{t_{k}} d s
\end{aligned}
$$

In a similar way to the proof of Theorem 4.3 , it can be shown that as $|\pi| \rightarrow 0$,

$$
\begin{aligned}
I_{1}^{\pi} \rightarrow & \int_{0}^{t} \frac{\partial f}{\partial x}\left(s, \xi_{s}, \eta_{s}\right) F_{s} d B_{s}^{H}+\int_{0}^{t} \frac{\partial^{2} f}{\partial x^{2}}\left(s, \xi_{s}, \eta_{s}\right) D_{s}^{\phi} \xi_{s} F_{s} d s \\
& +\int_{0}^{t} \frac{\partial^{2} f}{\partial x \partial y}\left(s, \xi_{s}, \eta_{s}\right) D_{s}^{\phi} \eta_{s} F_{s} d s
\end{aligned}
$$

and

$$
\begin{aligned}
I_{2}^{\pi} \rightarrow & \int_{0}^{t} \frac{\partial f}{\partial y}\left(s, \xi_{s}, \eta_{s}\right) G_{s} d B_{s}^{H}+\int_{0}^{t} \frac{\partial^{2} f}{\partial y^{2}}\left(s, \xi_{s}, \eta_{s}\right) D_{s}^{\phi} \eta_{s} G_{s} d s \\
& +\int_{0}^{t} \frac{\partial^{2} f}{\partial x \partial y}\left(s, \xi_{s}, \eta_{s}\right) D_{s}^{\phi} \xi_{s} G_{s} d s
\end{aligned}
$$

in $L^{2}$ proving the theorem.

The Itô formula for the Stratonovich type integrals is simpler.

Theorem 4.7 Let $\left(F_{t}, t \in[0, T]\right)$ be a process such that the assumptions of Theorem 4.3 are satisfied. Let $\xi_{t}=\int_{0}^{t} F_{s} \delta B_{s}^{H}$. Let $g: \mathbb{R}_{+} \times \mathbb{R} \rightarrow \mathbb{R}$ be a a twice continuously differentiable function with bounded derivatives to second order. Let $\left(\frac{\partial g}{\partial x}\left(s, \xi_{s}\right) F_{s}, s \in[0, T]\right)$ be in $\mathcal{L}(0, T)$. Then for $t \in[0, T]$

$$
g\left(t, \xi_{t}\right)=g(0,0)+\int_{0}^{t} \frac{\partial g}{\partial s}\left(s, \xi_{s}\right) d s+\int_{0}^{t} \frac{\partial g}{\partial x}\left(s, \xi_{s}\right) F_{s} \delta B_{s}^{H} \quad \text { a.s. }
$$


Proof Note that $\tilde{\xi}_{t}=\int_{0}^{t} F_{s} d B_{s}^{H}$ also exists and

$$
\xi_{t}=\tilde{\xi}_{t}+\int_{0}^{t} D_{s}^{\phi} F_{s} d s .
$$

Using the Itô formula (4.2)

$$
\begin{aligned}
g\left(t, \xi_{t}\right)= & g\left(t, \tilde{\xi}_{t}+\int_{0}^{t} D_{s}^{\phi} F_{s} d s\right) \\
= & g(0,0)+\int_{0}^{t} \frac{\partial g}{\partial s}\left(s, \xi_{s}\right) d s+\int_{0}^{t} \frac{\partial g}{\partial x}\left(s, \xi_{s}\right) D_{s}^{\phi} F_{s} d s \\
& +\int_{0}^{t} \frac{\partial g}{\partial x}\left(s, \xi_{s}\right) F_{s} d B_{s}^{H}+\int_{0}^{t} \frac{\partial^{2} g}{\partial x^{2}}\left(s, \xi_{s}\right) D_{s}^{\phi} \xi_{s} F_{s} d s
\end{aligned}
$$

Now

$$
\begin{aligned}
\int_{0}^{t} \frac{\partial g}{\partial x}\left(s, \xi_{s}\right) F_{s} \delta B_{s}^{H}= & \int_{0}^{t} g_{x}\left(s, \xi_{s}\right) F_{s} d B_{s}^{H}-\int_{0}^{t} D_{s}^{\phi}\left(g_{x}\left(s, \xi_{s}\right) F_{s}\right) d s \\
= & \int_{0}^{t} g_{x}\left(s, \xi_{s}\right) F_{s} \delta B_{s}^{H}-\int_{0}^{t} g_{x x}\left(s, \xi_{s}\right) D_{s}^{\phi} \xi_{s} F_{s} d s \\
& -\int_{0}^{t} g_{x}\left(s, \xi_{s}\right) D_{s}^{\phi} F_{s} d s
\end{aligned}
$$

Combining the above two equalities, it follows that

$$
g\left(t, \xi_{t}\right)=g(0,0)+\int_{0}^{t} \frac{\partial g}{\partial s}\left(s, \xi_{s}\right) d s+\int_{0}^{t} \frac{\partial g}{\partial x}\left(s, \xi_{s}\right) F_{s} \delta B_{s}^{H}
$$

proving the theorem.

Remark 1 The equation (4.4) can be expressed formally as

$$
\delta g\left(t, \xi_{t}\right)=g_{t}\left(t, \xi_{t}\right) d t+g_{x}\left(t, \xi_{t}\right) \delta \xi_{t}
$$

or more generally

$$
\begin{gathered}
\delta g\left(t, \xi_{t}^{1}, \xi_{t}^{2}, \cdots, \xi_{t}^{n}\right)=\frac{\partial g}{\partial t}\left(t, \xi_{t}^{1}, \xi_{t}^{2}, \cdots, \xi_{t}^{n}\right) d t+\frac{\partial g}{\partial x_{1}}\left(t, \xi_{t}^{1}, \xi_{t}^{2}, \cdots, \xi_{t}^{n}\right) \delta \xi_{t}^{1} \\
+\cdots+\frac{\partial g}{\partial x_{n}}\left(t, \xi_{t}^{1}, \xi_{t}^{2}, \cdots, \xi_{t}^{n}\right) \delta \xi_{t}^{n}
\end{gathered}
$$

\section{Two Applications of the Itô Formula}

Two applications are given now of the Itô formula for fractional Brownian motion. First, the so-called homogeneous chaos is extended to a fractional Brownian motion. Second, an $L^{p}$ estimate of the (Itô type) stochastic integral for a fractional Brownian motion is given. 
Let $H_{n}(x)$ be the Hermite polynomial of degree $n$, that is,

$$
e^{t x-\frac{1}{2} t^{2}}=\sum_{n=0}^{\infty} t^{n} H_{n}(x)
$$

Let

$$
|f|_{\phi, t}=\left\{\int_{0}^{t} \int_{0}^{t} \phi(u, v) f_{u} f_{v} d u d v\right\}^{1 / 2}
$$

Define

$$
\tilde{f}_{t}=|f|_{\phi, t}^{-1} \int_{0}^{t} f_{s} d B_{s}^{H}
$$

and

$$
H_{n}^{\phi, f}(t)=|f|_{\phi, t}^{n} H_{n}\left(\tilde{f}_{t}\right)
$$

Theorem 5.1 If $f 1_{[0, T]} \in L_{\phi}^{2}$, then the following equality is satisfied

$$
d H_{n}^{\phi, f}(t)=n H_{n-1}^{\phi, f}(t) f_{t} d B_{t}^{H}
$$

where $d$ is the Itô type differential given in Theorem 4.3 and $t \in[0, T]$.

Proof Fix $n$ and denote $X_{t}=H_{n}^{\phi, f}(t)$ for $t \in[0, T]$. Using the Itô formula (Theorem 4.3) and prime for differentiation it follows that

$$
\begin{aligned}
d X_{t}= & n|f|_{\phi, t}^{n-2} f_{t} \int_{0}^{t} \phi(u, t) f_{u} d u H_{n}\left(\tilde{f}_{t}\right) d t \\
& -|f|_{\phi, t}^{n} f_{t} \int_{0}^{t} \phi(u, t) f_{u} d u H_{n}^{\prime}\left(\tilde{f}_{t}\right)|f|_{\phi, t}^{-3}\left(\int_{0}^{t} f_{s} d B_{s}^{H}\right) d t \\
& +|f|_{\phi, t}^{n} H_{n}^{\prime}\left(\tilde{f}_{t}\right)|f|_{\phi, t}^{-1} f_{t} d B_{t}^{H} \\
& +|f|_{\phi, t}^{n} f_{t} \int_{0}^{t} \phi(u, t) f_{u} d u H_{n}^{\prime \prime}\left(\tilde{f}_{t}\right)|f|_{\phi, t}^{-2} d t \\
= & n|f|_{\phi, t}^{n-2} H_{n-1}\left(\tilde{f}_{t}\right) f_{t} d B_{t}^{H} \\
& +|f|_{\phi, t}^{n-2} f_{t} \int_{0}^{t} \phi(u, t) f_{u} d u \\
& \cdot\left\{n H_{n}\left(\tilde{f}_{t}\right)-\tilde{f}_{t} H_{n}^{\prime}\left(\tilde{f}_{t}\right)+H_{n}^{\prime \prime}\left(\tilde{f}_{t}\right)\right\} d t
\end{aligned}
$$

It is well known that for each $n \in \mathbb{N}$ the Hermite polynomial satisfies

$$
n H_{n}(x)-x H_{n}^{\prime}(x)+H_{n}^{\prime \prime}(x)=0
$$


for each $x \in \mathbb{R}$. Thus the sum of the terms in the above \{\} equals 0 . The first term is

$$
n H_{n-1}^{\phi, f}(t) f_{t} d B_{t}^{H} .
$$

Thus

$$
d H_{n}^{\phi, f}(t)=n H_{n-1}^{\phi, f}(t) f_{t} d B_{t}^{H}
$$

proving the theorem.

The following estimate for the $L^{p}$ norm is useful in some applications.

Theorem 5.2 Let $\left(g_{s}, s \in[0, t]\right)$ be a stochastic process satisfying the assumptions of Theorem 4.3 for $F$. Let $F_{t}:=\int_{0}^{t} g_{s} d B_{s}^{H}$. If $\mathbb{E} \int_{0}^{t}\left|g_{s}\right|^{p} d s<\infty, \int_{0}^{t} \mathbb{E}\left|D_{s}^{\phi} F_{s}\right|^{p} d s<\infty$ and $F^{p-1} g \in \mathcal{L}(0, t)$, then

$$
\mathbb{E} F_{t}^{p} \leq p^{p}\left\{\int_{0}^{t}\left(\mathbb{E}\left|g_{s} D_{s}^{\phi} F_{s}\right|^{p / 2}\right)^{2 / p} d s\right\}^{p / 2} .
$$

Proof Applying the Itô formula (Theorem 4.3) to $F_{t}^{p}$ (by the assumption that $F^{p-1} g \in$ $\mathcal{L}(0, t)$, the restriction on the boundedness of $f$ to its second derivatives in Theorem 4.3 can be removed), it follows that

$$
F_{t}^{p}=p \int_{0}^{t} F_{s}^{p-1} g_{s} d B_{s}^{H}+p(p-1) \int_{0}^{t} F_{s}^{p-2} g_{s} D_{s}^{\phi} F_{s} d s .
$$

Thus

$$
\begin{aligned}
\mathbb{E} F_{t}^{p} & =p(p-1) \int_{0}^{t} \mathbb{E}\left(F_{s}^{p-2} g_{s} D_{s}^{\phi} F_{s}\right) d s . \\
\mathbb{E} F_{t}^{p} & \leq p(p-1) \int_{0}^{t} \mathbb{E}\left|F_{s}^{p-2} g_{s} D_{s}^{\phi} F_{s}\right| d s \\
& \leq p^{2} \int_{0}^{t}\left(\mathbb{E} F_{s}^{p}\right)^{\frac{p-2}{p}}\left(\mathbb{E}\left|g_{s} D_{s}^{\phi} F_{s}\right|^{\frac{p}{2}}\right)^{\frac{2}{p}} d s
\end{aligned}
$$

By an inequality of Langenhop, (e.g. [1]), there is the inequality

$$
\mathbb{E} F_{t}^{p} \leq p^{p}\left\{\int_{0}^{t}\left(\mathbb{E}\left|g_{s} D_{s}^{\phi} F_{s}\right|^{\frac{p}{2}}\right)^{\frac{2}{p}} d s\right\}^{\frac{p}{2}} .
$$

This completes the proof of the theorem.

Corollary 5.3 Let the conditions of Theorem 5.2 be satisfied and let $p \geq 2$. Then

$$
\mathbb{E} F_{t}^{p} \leq p^{p}\left\{\int_{0}^{t}\left(\mathbb{E}\left|g_{s}\right|^{p}\right)^{\frac{2}{p}} d s+\int_{0}^{t}\left(\mathbb{E}\left|D_{s}^{\phi} F_{s}\right|^{p}\right)^{\frac{2}{p}} d s\right\}^{\frac{p}{2}} .
$$


Proof From $|a b| \leq a^{2}+b^{2}$, it follows that

$$
\mathbb{E}\left|g_{s} D_{s}^{\phi} F_{s}\right|^{\frac{p}{2}} \leq \mathbb{E}\left|g_{s}\right|^{p}+\mathbb{E}\left|D_{s}^{\phi} F_{s}\right|^{p}
$$

Thus

$$
\begin{aligned}
\left(\mathbb{E}\left|g_{s} D_{s}^{\phi} F_{s}\right|^{\frac{p}{2}}\right)^{\frac{2}{p}} & \leq\left(\mathbb{E}\left|g_{s}\right|^{p}+\mathbb{E}\left|D_{s}^{\phi} F_{s}\right|^{p}\right)^{\frac{2}{p}} \\
& \leq\left(\mathbb{E}\left|g_{s}\right|^{p}\right)^{\frac{2}{p}}+\left(\mathbb{E}\left|D_{s}^{\phi} F_{s}\right|^{p}\right)^{\frac{2}{p}}
\end{aligned}
$$

This verifies the corollary.

\section{Iterated Integrals and Multiple Integrals}

Let $f \in L_{\phi}^{2}\left(\mathbb{R}_{+}\right)$be such that $|f|_{\phi}=1$. Similar to [8] define $\left(\int_{0}^{\infty} f_{s} d B_{s}^{H}\right)^{\diamond n}$ as the $n$-th Wick power of $\int_{0}^{\infty} f_{s} d B_{s}^{H}$, that is, denote formally

$$
\begin{gathered}
\left(\int_{0}^{\infty} f_{s} d B_{s}^{H}\right)^{\diamond n}:=\left(\int_{0}^{\infty} f_{s} d B_{s}^{H}\right)^{\diamond(n-1)} \diamond \int_{0}^{\infty} f_{s} d B_{s}^{H}, \quad n=2,3, \cdots \\
\exp ^{\diamond}\left(\int_{0}^{\infty} f_{s} d B_{s}^{H}\right):=\sum_{n=0}^{\infty} \frac{1}{n !}\left(\int_{0}^{\infty} f_{s} d B_{s}^{H}\right)^{\diamond n} ; \\
\log ^{\diamond}\left(1+\int_{0}^{\infty} f_{s} d B_{s}^{H}\right):=\sum_{n=1}^{\infty} \frac{(-1)^{n-1}}{n}\left(\int_{0}^{\infty} f_{s} d B_{s}^{H}\right)^{\diamond n}
\end{gathered}
$$

Lemma 6.1 If $|f|_{\phi}=1$, then $\left(\int_{0}^{\infty} f_{s} d B_{s}^{H}\right)^{\diamond n}$ is well defined for each $n \in \mathbb{N}$ and

$$
\left(\int_{0}^{\infty} f_{s} d B_{s}^{H}\right)^{\diamond n}=H_{n}\left(\int_{0}^{\infty} f_{s} d B_{s}^{H}\right)
$$

where $H_{n}$ denotes the Hermite polynomial of degree $n$.

Proof The equality (6.1) is verified by induction.

It is easy to see that (6.1) is true for $n=1$. Let (6.1) be true for $1,2, \cdots, n-1$. Then

$$
\begin{aligned}
\left(\int_{0}^{\infty} f_{s} d B_{s}^{H}\right)^{\diamond n} & =H_{n-1}\left(\int_{0}^{\infty} f_{s} d B_{s}^{H}\right) \diamond \int_{0}^{\infty} f_{s} d B_{s}^{H} \\
& =H_{n-1}\left(\int_{0}^{\infty} f_{s} d B_{s}^{H}\right) \int_{0}^{\infty} f_{s} d B_{s}^{H}-D_{\Phi f}\left\{H_{n-1}\left(\int_{0}^{\infty} f_{s} d B_{s}^{H}\right)\right\} \\
& =H_{n-1}\left(\int_{0}^{\infty} f_{s} d B_{s}^{H}\right) \int_{0}^{\infty} f_{s} d B_{s}^{H}-H_{n-1}^{\prime}\left(\int_{0}^{\infty} f_{s} d B_{s}^{H}\right)|f|_{\phi}^{2} \\
& =H_{n-1}\left(\int_{0}^{\infty} f_{s} d B_{s}^{H}\right) \int_{0}^{\infty} f_{s} d B_{s}^{H}-H_{n-1}^{\prime}\left(\int_{0}^{\infty} f_{s} d B_{s}^{H}\right) \\
& =H_{n}\left(\int_{0}^{\infty} f_{s} d B_{s}^{H}\right)
\end{aligned}
$$


by an identity for Hermite polynomials. This verifies the equation (6.1).

For an arbitrary, nonzero $f \in L_{\phi}^{2}\left(\mathbb{R}_{+}\right)$, the product defined in (6.1) is extended as

$$
\left(\int_{0}^{\infty} f_{s} d B_{s}^{H}\right)^{\diamond n}=|f|_{\phi}^{n}\left(\frac{\int_{0}^{\infty} f_{s} d B_{s}^{H}}{|f|_{\phi}}\right)^{\diamond n}=|f|_{\phi}^{n} H_{n}\left(\int_{0}^{\infty} \frac{\int_{0}^{\infty} f_{s} d B_{s}^{H}}{|f|_{\phi}}\right) .
$$

Lemma 6.2 If $f \in L_{\phi}^{2}\left(\mathbb{R}_{+}\right)$, then $\left(\int_{0}^{\infty} f_{s} d B_{s}^{H}\right)^{\diamond n}$ is well defined for each $n \in \mathbb{N}$ and

$$
\left(\int_{0}^{t} f_{s} d B_{s}^{H}\right)^{\diamond n}=H_{n}^{\phi, f}(t)
$$

Since $\int_{0}^{\infty} f_{s} d B_{s}^{H}$ is a Gaussian random variable, it is easy to estimate its moments and to show that the series defining $\exp ^{\diamond}\left(\int_{0}^{\infty} f_{s} d B_{s}^{H}\right)$ is convergent in $L^{2}(\Omega, \mathcal{F}, P)$. Moreover there is the following corollary.

Corollary 6.3 If $f \in L_{\phi}^{2}\left(\mathbb{R}_{+}\right)$, then

$$
\exp ^{\diamond}\left(\int_{0}^{\infty} f_{s} d B_{s}^{H}\right)=\varepsilon(f)=\exp \left(\int_{0}^{\infty} f_{s} d B_{s}^{H}-\frac{1}{2}|f|_{\phi}^{2}\right) .
$$

Proof It follows that

$$
\begin{aligned}
\exp ^{\diamond}\left(\int_{0}^{\infty} f_{s} d B_{s}^{H}\right) & =\sum_{n=0}^{\infty} \frac{1}{n !}\left(\int_{0}^{\infty} f_{s} d B_{s}^{H}\right)^{\diamond n} \\
& =\sum_{n=0}^{\infty} \frac{1}{n !}|f|_{\phi}^{n} H_{n}\left(\frac{\int_{0}^{\infty} f_{s} d B_{s}^{H}}{|f|_{\phi}}\right) \\
& =\exp \left(|f|_{\phi} \frac{\int_{0}^{\infty} f_{s} d B_{s}^{H}}{|f|_{\phi}}-\frac{1}{2}|f|_{\phi}^{2}\right) \\
& =\exp \left(\int_{0}^{\infty} f_{s} d B_{s}^{H}-\frac{1}{2}|f|_{\phi}^{2}\right)
\end{aligned}
$$

This completes the proof of the lemma.

The following lemma is also easy to prove.

Lemma 6.4 For any two functions $f$ and $g$ in $L_{\phi}^{2}\left(\mathbb{R}_{+}\right)$with $\langle f, g\rangle_{\phi}=0$, the following equality is satisfied

$$
\left(\int_{0}^{\infty} f_{s} d B_{s}^{H}\right)^{\diamond n} \diamond\left(\int_{0}^{\infty} g_{s} d B_{s}^{H}\right)^{\diamond m}=\left(\int_{0}^{\infty} f_{s} d B_{s}^{H}\right)^{\diamond n}\left(\int_{0}^{\infty} g_{s} d B_{s}^{H}\right)^{\diamond m}=H_{n}^{\phi, f}(\infty) H_{m}^{\phi, g}(\infty) .
$$

Since $\int_{0}^{\infty} f_{s} d B_{s}^{H} /|f|_{\phi}$ and $\int_{0}^{\infty} g_{s} d B_{s}^{H} /|g|_{\phi}$ are Gaussian random variables with mean 0 and variance 1 , their covariance is

$$
\mathbb{E}\left\{\left(\int_{0}^{\infty} f_{s} d B_{s}^{H} /|f|_{\phi}\right)\left(\int_{0}^{\infty} g_{s} d B_{s}^{H} /|g|_{\phi}\right)\right\}=\left\langle f /|f|_{\phi}, g /|g|_{\phi}\right\rangle_{\phi},
$$


It follows that

$$
\begin{aligned}
& \mathbb{E}\left\{\left(\int_{0}^{\infty} f_{s} d B_{s}^{H}\right)^{\diamond n}\left(\int_{0}^{\infty} g_{s} d B_{s}^{H}\right)^{\diamond m}\right\} \\
= & \mathbb{E}\left\{|f|_{\phi}^{n}|g|_{\phi}^{m} H_{n}\left(\int_{0}^{\infty} f_{s} d B_{s}^{H} /|f|_{\phi}\right) H_{m}\left(\int_{0}^{\infty} g_{s} d B_{s}^{H} /|g|_{\phi}\right)\right\} \\
= & \begin{cases}0 & \text { if } m \neq n \\
|f|_{\phi}^{n}|g|_{\phi}^{n}\left\langle f /|f|_{\phi}, g /|g|_{\phi}\right\rangle_{\phi}^{n} & \text { if } m=n\end{cases} \\
= & \begin{cases}0 & \text { if } m \neq n \\
\langle f, g\rangle_{\phi}^{n} & \text { if } m=n\end{cases}
\end{aligned}
$$

By a polarization technique [21] it is easy to verify the following lemma.

Lemma 6.5 Let $f^{1}, \cdots, f^{n}, g^{1}, \cdots, g^{m} \in L_{\phi}^{2}\left(\mathbb{R}_{+}\right)$. The following equality is satisfied

$$
\begin{aligned}
& \mathbb{E}\left\{\left(\int_{0}^{\infty} f_{s}^{1} d B_{s}^{H} \diamond \cdots \diamond \int_{0}^{\infty} f_{s}^{n} d B_{s}^{H}\right)\left(\int_{0}^{\infty} g_{s}^{1} d B_{s}^{H} \diamond \cdots \diamond \int_{0}^{\infty} g_{s}^{m} d B_{s}^{H}\right)\right\} \\
= & \begin{cases}0 & \text { if } n \neq m \\
\frac{1}{n !} \sum_{\sigma}\left\langle f^{1}, g^{\sigma(1)}\right\rangle_{\phi}\left\langle f^{2}, g^{\sigma(2)}\right\rangle_{\phi} \cdots\left\langle f^{n}, g^{\sigma(n)}\right\rangle_{\phi} & \text { if } n=m\end{cases}
\end{aligned}
$$

where $\sum_{\sigma}$ denotes the sum over all permutations $\sigma$ of $\{1,2, \cdots, n\}$.

Let $e_{1}, e_{2}, \cdots, e_{n}, \cdots$ be a complete orthonormal basis of $L_{\phi}^{2}\left(\mathbb{R}_{+}\right)$. Consider the $n$-th symmetric tensor product of $L_{\phi}^{2}\left(\mathbb{R}_{+}\right): L_{\phi}^{s}\left(\mathbb{R}_{+}^{n}\right):=L_{\phi}^{2}\left(\mathbb{R}_{+}\right) \otimes \cdots \otimes L_{\phi}^{2}\left(\mathbb{R}_{+}\right)$. It is the completion of all functions of the following form

$$
f\left(s_{1}, \cdots, s_{n}\right)=\sum_{1 \leq k_{1}, \cdots, k_{n} \leq k} a_{k_{1} \cdots k_{n}} e_{k_{1}}\left(s_{1}\right) e_{k_{2}}\left(s_{2}\right) \cdots e_{k_{n}}\left(s_{n}\right),
$$

where $f$ is a symmetric function of its variables $s_{1}, \cdots, s_{n}$ and $k$ is a positive integer. The set of all of the above finite sums is denoted $\mathcal{L}_{n}$. For an element of the form (6.2), its multiple integral is defined by

$$
I_{n}(f)=\sum_{1 \leq k_{1}, \cdots, k_{n} \leq k} a_{k_{1}} \cdots k_{n} \int_{0}^{\infty} e_{k_{1}}(s) d B_{s}^{H} \diamond \int_{0}^{\infty} e_{k_{2}}(s) d B_{s}^{H} \diamond \cdots \diamond \int_{0}^{\infty} e_{k_{n}}(s) d B_{s}^{H}
$$

By Lemma 6.5, the norm of (6.3) is given by

$$
\begin{gathered}
\mathbb{E}\left|I_{n}(f)\right|^{2}=\int_{\mathbb{R}_{+}^{2 n}} \phi\left(u_{1}, v_{1}\right) \phi\left(u_{2}, v_{2}\right) \cdots \phi\left(u_{n}, v_{n}\right) f\left(u_{1}, u_{2}, \cdots, u_{n}\right) \\
f\left(v_{1}, v_{2}, \cdots, v_{n}\right) d u_{1} d u_{2} \cdots d u_{n} d v_{1} d v_{2} \cdots d v_{n} .
\end{gathered}
$$


Thus for any element $f$ in

$L_{\phi}^{2}\left(\mathbb{R}_{+}^{n}\right)=\left\{f: \mathbb{R}_{+}^{n} \rightarrow \mathbb{R} ; f\right.$ is symmetric with respect to its arguments, $\left.|f|_{\phi}^{2}:=\langle f, f\rangle_{\phi}<\infty\right\}$

where

$$
\begin{array}{r}
\langle f, g\rangle_{\phi}=\int_{\mathbb{R}_{+}^{2 n}} \phi\left(u_{1}, v_{1}\right) \phi\left(u_{2}, v_{2}\right) \cdots \phi\left(u_{n}, v_{n}\right) f\left(u_{1}, u_{2}, \cdots, u_{n}\right) \\
g\left(v_{1}, v_{2}, \cdots, v_{n}\right) d u_{1} d u_{2} \cdots d u_{n} d v_{1} d v_{2} \cdots d v_{n} .
\end{array}
$$

The multiple integral $I_{n}(f)$ can be defined by a limit from elements in $\mathcal{L}_{n}$ and it follows that

$$
\mathbb{E}\left(\left|I_{n}(f)\right|^{2}\right)=|f|_{\phi}^{2} .
$$

The following lemma can also be shown by the polarization technique.

Lemma 6.6 If $f \in L_{\phi}^{2}\left(\mathbb{R}_{+}^{n}\right)$ and $g \in L_{\phi}^{2}\left(\mathbb{R}_{+}^{m}\right)$, then

$$
\mathbb{E}\left(I_{n}(f) I_{m}(g)\right)= \begin{cases}\langle f, g\rangle_{\phi} & \text { if } n=m \\ 0 & \text { if } n \neq m\end{cases}
$$

Let $f \in L_{\phi}^{2}\left(\mathbb{R}_{+}^{n}\right)$. The iterated integral can be defined by the recursive formula

$$
\begin{aligned}
& \int_{0 \leq s_{1}<s_{2}<\cdots<s_{n} \leq t} f\left(s_{1}, s_{2}, \cdots, s_{n}\right) d B_{s_{1}}^{H} d B_{s_{2}}^{H} \cdots d B_{s_{n}}^{H} \\
& =\int_{0}^{t}\left(\int_{0 \leq s_{1}<s_{2}<\cdots \leq s_{n}} f\left(s_{1}, s_{2}, \cdots, s_{n-1}, s_{n}\right) d B_{s_{1}}^{H} d B_{s_{2}}^{H} \cdots d B_{s_{n-1}}^{H}\right) d B_{s_{n}}^{H}
\end{aligned}
$$

Theorem 6.7 If $f \in L_{\phi}^{2}\left(\mathbb{R}_{+}^{n}\right)$, then the iterated integral (6.6) exists and

$$
I_{n}(f)=n ! \int_{0 \leq s_{1}<s_{2}<\cdots<s_{n} \leq t} f\left(s_{1}, s_{2}, \cdots, s_{n}\right) d B_{s_{1}}^{H} d B_{s_{2}}^{H} \cdots d B_{s_{n}}^{H} .
$$

Proof First let $f$ have the special form $f=g^{\otimes n}$, that is, $f\left(s_{1}, s_{2}, \cdots, s_{n}\right)=g\left(s_{1}\right) g_{2}\left(s_{2}\right) \cdots g\left(s_{n}\right)$. Then

$$
I_{n}(f)=H_{n}^{\phi, g}(t)
$$

and

$$
\begin{aligned}
d I_{n}(f) & =d H_{n}^{\phi, g}(t) \\
& =n H_{n-1}^{\phi, g}(t) g(t) d B_{t}^{H} \\
& =n I_{n-1}\left(g^{\otimes(n-1)}\right) g(t) d B_{t}^{H}
\end{aligned}
$$

This verifies (6.7) for the case where $f=g^{\otimes n}$. By the polarization technique [21], the theorem follows easily. 
Remark 2 For Brownian motion, a multiple integral was originally introduced by Wiener [24]; Wiener's original multiple integral is in fact a multiple integral of Stratonovich type. The multiple integral of Itô type was introduced in [16].

For Brownian motion, the multiple Stratonovich integrals also have been widely used in the applications. Since the work of [9] and [10], it is known that the definition of multiple Stratonovich integrals is related to the definition of "trace". There has been much work on this topic. The reader is referred to [13] and the references therein.

A class of traces and multiple Stratonovich integrals are defined and the Hu-Meyer formula is extended to the fractional Brownian motions.

As in [11], introduce the $\phi$-trace $\operatorname{Tr}_{\phi}$ for simple functions. This new type of trace extends the classical one and plays an important role in this section. form

Let $f_{1}, f_{2}, \cdots, f_{m} \in L_{\phi}^{2}\left(\mathbb{R}_{+}\right)$. Consider the simple functions in $L_{\phi}^{2}\left(\mathbb{R}_{+}^{n}\right)$ of the following

$$
f\left(t_{1}, t_{2}, \cdots, t_{n}\right)=\sum_{1 \leq i_{1}, i_{2}, \cdots, i_{n} \leq m} a_{i_{1}, i_{2}, \cdots, i_{n}} f_{i_{1}}\left(t_{1}\right) f_{i_{2}}\left(t_{2}\right) \cdots f_{i_{n}}\left(t_{n}\right) .
$$

If $f$ is given by $(6.8)$, then for $k \in\left\{1,2, \cdots,\left[\frac{n}{2}\right]\right\}$, define

$$
\begin{aligned}
\operatorname{Tr}_{\phi}^{k} f\left(t_{1}, \cdots, t_{n-2 k}\right)=\int_{0}^{\infty} & \cdots \int_{0}^{\infty} f\left(s_{1}, s_{2}, \cdots, s_{2 k-1}, s_{2 k}, t_{1}, \cdots, t_{n-2 k}\right) \\
& \phi\left(s_{1}, s_{2}\right) \phi\left(s_{3}, s_{4}\right) \cdots \phi\left(s_{2 k-1}, s_{2 k}\right) d s_{1} \cdots d s_{2 k} .
\end{aligned}
$$

To define the trace for general functions, as in [11], let $\gamma_{\varepsilon}(s, t)$ be an approximation of the Dirac function, that is,

$$
\lim _{\varepsilon \rightarrow 0} \int \gamma_{\varepsilon}(s, t) f(s) d s=f(t)
$$

in some sense. Assume that

$$
\int_{0}^{\infty} \int_{0}^{\infty} \gamma_{\varepsilon}(s, t)^{2} d s d t<\infty
$$

For any function $f \in L_{\phi}^{2}\left(\mathbb{R}_{+}^{n}\right)$, the following approximation

$$
f^{\varepsilon}\left(t_{1}, t_{2}, \cdots, t_{n}\right)=\int_{0}^{\infty} \cdots \int_{0}^{\infty} f\left(s_{1}, s_{2}, \cdots, s_{n}\right) \gamma_{\varepsilon}\left(s_{1}, t_{1}\right) \gamma_{\varepsilon}\left(s_{2}, t_{2}\right) \cdots \gamma_{\varepsilon}\left(s_{n}, t_{n}\right) d s_{1} d s_{2} \cdots d s_{n}
$$

is a simple function of type (6.8) and if $f$ is symmetric, then $f^{\varepsilon}$ is also symmetric. Let

$$
\rho_{\varepsilon}(s, t)=\int_{0}^{\infty} \gamma_{\varepsilon}(s, u) \gamma_{\varepsilon}(t, u) d u .
$$

According to the definition of $f^{\varepsilon}$,

$$
\begin{aligned}
\operatorname{Tr}_{\phi}^{k} f^{\varepsilon}\left(t_{1}, \cdots, t_{n-2 k}\right)=\int_{0}^{\infty} & \cdots \int_{0}^{\infty} f\left(s_{1}, s_{2}, \cdots, s_{n}\right) \rho\left(s_{1}, s_{2}\right) \cdots \rho\left(s_{2 k-1}, s_{2 k}\right) \\
& \gamma_{\varepsilon}\left(s_{2 k+1}, t_{1}\right) \cdots \gamma_{\varepsilon}\left(s_{n}, t_{n-2 k}\right) d s_{1} d s_{2} \cdots d s_{n}
\end{aligned}
$$


Definition 6.1 Let $f \in L_{\phi}^{2}\left(\mathbb{R}_{+}^{n}\right)$. The $k$-th trace of $f$ is said to exist if $\operatorname{Tr}_{\phi}^{k} f^{\varepsilon}\left(t_{1}, \cdots, t_{n-2 k}\right)$ converges to a function in $L_{\phi}^{2}\left(\mathbb{R}_{+}^{n-2 k}\right)$ as $\varepsilon \rightarrow 0$. The limiting function is called the $k$-th trace of $f$, that is,

$$
\operatorname{Tr}_{\phi}^{k} f\left(t_{1}, \cdots, t_{n-2 k}\right)=\lim _{\varepsilon \rightarrow 0} \operatorname{Tr}_{\phi}^{k} f^{\varepsilon}\left(t_{1}, \cdots, t_{n-2 k}\right) .
$$

Now introduce the multiple Stratonovich integrals for fractional Brownian motions. Define $\left(B_{t}^{H}\right)^{\varepsilon}=\int_{0}^{\infty} \gamma_{\varepsilon}(t, s) d B_{s}^{H}$. Then $\left(B_{t}^{H}\right)^{\varepsilon}$ is differentiable. Let $f \in L_{\phi}^{2}\left(\mathbb{R}_{+}^{n}\right)$. Consider

$$
S_{n}^{\varepsilon}(f):=\int_{\mathbb{R}_{+}^{n}} f\left(s_{1}, s_{2}, \cdots, s_{n}\right)\left(\dot{B}_{s_{1}}^{H}\right)^{\varepsilon}\left(\dot{B}_{s_{2}}^{H}\right)^{\varepsilon} \cdots\left(\dot{B}_{s_{n}}^{H}\right)^{\varepsilon} d s_{1} d s_{2} \cdots d s_{n} .
$$

Definition 6.2 If $S_{n}^{\varepsilon}(f)$ converges in $L^{2}(\Omega, \mathcal{F}, P)$ as $\varepsilon \rightarrow 0$, then the multiple Stratonovich integral is said to exist and is denoted by

$$
S_{n}(f)=\int_{\mathbb{R}_{+}^{n}} f\left(s_{1}, s_{2}, \cdots, s_{n}\right) \delta B_{s_{1}}^{H} \delta B_{s_{2}}^{H} \cdots \delta B_{s_{n}}^{H} .
$$

The remaining part of this section is devoted to giving conditions such that $S_{n}^{\varepsilon}(f)$ is convergent in $L^{2}(\Omega, \mathcal{F}, P)$.

By the identity $x^{n}=\sum_{k \leq\left[\frac{n}{2}\right]} \frac{n !}{2^{k} k !(n-2 k) !} H_{n-2 k}(x)$, it follows that

$$
\begin{aligned}
\left(\int_{0}^{\infty} f_{s} d B_{s}^{H}\right)^{n} & =|f|_{\phi}^{n}\left(\frac{\int_{0}^{\infty} f_{s} d B_{s}^{H}}{|f|_{\phi}}\right)^{n} \\
& =|f|_{\phi}^{n} \sum_{k \leq\left[\frac{n}{2}\right]} \frac{n !}{2^{k} k !(n-2 k) !} H_{n-2 k}\left(\frac{\int_{0}^{\infty} f_{s} d B_{s}^{H}}{|f|_{\phi}}\right) \\
& =\sum_{k \leq\left[\frac{n}{2}\right]} \frac{n !}{2^{k} k !(n-2 k) !}|f|_{\phi}^{n}\left(\frac{\int_{0}^{\infty} f_{s} d B_{s}^{H}}{|f|_{\phi}}\right)^{\diamond(n-2 k)} \\
& =\sum_{k \leq\left[\frac{n}{2}\right]} \frac{n !}{2^{k} k !(n-2 k) !}|f|_{\phi}^{2 k}\left(\int_{0}^{\infty} f_{s} d B_{s}^{H}\right)^{\diamond(n-2 k)} \\
& =\sum_{k \leq\left[\frac{n}{2}\right]} \frac{n !}{2^{k} k !(n-2 k) !}|f|_{\phi}^{2 k} I_{n-2 k}\left(f^{\otimes(n-2 k)}\right) \\
& =\sum_{k \leq\left[\frac{n}{2}\right]} \frac{n !}{2^{k} k !(n-2 k) !} I_{n-2 k}\left(\operatorname{Tr}_{\phi}^{k}\left(f^{\otimes n}\right)\right)
\end{aligned}
$$

where $f^{\otimes n}$ is the symmetric tensor product of $f$, that is, $f^{\otimes n}\left(s_{1}, s_{2}, \cdots, s_{n}\right)=f\left(s_{1}\right) f\left(s_{2}\right) \cdots f\left(s_{n}\right)$.

Let $f_{1}, f_{2}, \cdots, f_{n} \in L_{\phi}^{2}\left(\mathbb{R}_{+}\right)$and let $f$ be the symmetrization of $f_{1} f_{2} \cdots f_{n}$. Then by a polarization technique,

$$
\begin{aligned}
& \int_{0}^{\infty} f_{1}(s) d B_{s}^{H} \int_{0}^{\infty} f_{2}(s) d B_{s}^{H} \cdots \int_{0}^{\infty} f_{n}(s) d B_{s}^{H} \\
= & \sum_{k \leq\left[\frac{n}{2}\right]} \frac{n !}{2^{k} k !(n-2 k) !} I_{n-2 k}\left(\operatorname{Tr}_{\phi}^{k}(f)\right)
\end{aligned}
$$


Using this formula it follows that

$$
\begin{gathered}
S_{n}^{\varepsilon}(f)=\sum_{k \leq\left[\frac{n}{2}\right]} \frac{n !}{2^{k} k !(n-2 k) !} \int_{\mathbb{R}_{+}^{n}} f\left(s_{1}, s_{2}, \cdots, s_{n}\right) \rho_{\varepsilon}\left(s_{1}, s_{2}\right) \rho_{\varepsilon}\left(s_{3}, s_{4}\right) \cdots \rho_{\varepsilon}\left(s_{2 k-1}, s_{2 k}\right) \\
\gamma_{\varepsilon}\left(s_{2 k+1}, t_{1}\right) \cdots \gamma_{\varepsilon}\left(s_{n}, t_{n-2 k}\right) d s_{1} \cdots d s_{n} d B_{t_{1}}^{H} \cdots d B_{t_{n-2 k}}^{H}
\end{gathered}
$$

It is easy to verify the following result.

Theorem 6.8 Let $f \in L_{\phi}^{2}\left(\mathbb{R}_{+}^{n}\right)$ be such that all of the traces exist in the following sense: For $1 \leq k \leq\left[\frac{n}{2}\right]$

$$
\int_{\mathbb{R}_{+}^{n}} f\left(s_{1}, s_{2}, \cdots, s_{n}\right) \rho_{\varepsilon}\left(s_{1}, s_{2}\right) \rho_{\varepsilon}\left(s_{3}, s_{4}\right) \cdots \rho_{\varepsilon}\left(s_{2 k-1}, s_{2 k}\right) \gamma_{\varepsilon}\left(s_{2 k+1}, t_{1}\right) \cdots \gamma_{\varepsilon}\left(s_{n}, t_{n-2 k}\right) d s_{1} \cdots d s_{n}
$$

converges to a function $\operatorname{Tr}_{\phi}^{k} f$ in $L_{\phi}^{2}\left(\mathbb{R}_{+}^{n-2 k}\right)$ as $\varepsilon \rightarrow 0$. Then the sequence $\left(S_{n}^{\varepsilon}(f), n \in \mathbb{N}\right)$ converges in $L^{2}(\Omega, \mathcal{F}, P)$ and the limit is given by the extended Hu-Meyer formula

$$
S_{n}(f)=\sum_{k \leq\left[\frac{n}{2}\right]} \frac{n !}{2^{k} k !(n-2 k) !} I_{n-2 k}\left(\operatorname{Tr}_{\phi}^{k} f\right) .
$$

Remark 3 It should be noted that the analogue of this theorem and in particular the formula (6.10) has been discussed extensively for the Brownian motion.

As a consequence of Theorem 6.8, Equation (6.10), a chaos expansion theorem is described. It is well known that the family of all polynomials in the random variables $B_{t_{1}}^{H}, \cdots, B_{t_{k}}^{H}$, for $0 \leq t_{1}<\cdots<t_{k}$ and $k \in \mathbb{N}$ is dense in $L^{2}(\Omega, \mathcal{F}, P)$. Since each of these polynomials is a finite sum of the monomials of the form $\int_{0}^{\infty} f_{1}\left(s_{1}\right) \delta B_{s_{1}}^{H} \cdots \int_{0}^{\infty} f_{n}\left(s_{n}\right) \delta B_{s_{n}}^{H}$, where $f_{1}, \cdots, f_{n} \in L_{\phi}^{2}$. This product of integrals of Wiener type can be expressed as a multiple Stratonovich integral $\int_{0}^{\infty} \cdots \int_{0}^{\infty}\left(f_{1} \otimes \cdots \otimes f_{n}\right)\left(s_{1}, \cdots, s_{n}\right) \delta B_{s_{1}}^{H} \cdots \delta B_{s_{n}}^{H}$. By the equality (6.10), this multiple integral of Stratonovich type can be expressed as a finite, linear combination of multiple integrals of Itô type. Thus the family of all linear combinations of multiple integrals of the form $\int_{0}^{\infty} \cdots \int_{0}^{\infty}\left(f_{1} \otimes \cdots \otimes f_{n}\right)\left(s_{1}, \cdots, s_{n}\right) d B_{s_{1}}^{H} \cdots d B_{s_{n}}^{H}$ is dense in $L^{2}(\Omega, \mathcal{F}, P)$. Thus

$$
\begin{gathered}
L^{2}(\Omega, \mathcal{F}, P)=\left\{F: F=F_{0}+\sum_{n=1}^{\infty} \int_{0}^{\infty} \cdots \int_{0}^{\infty} f_{n}\left(s_{1}, \cdots, s_{n}\right) d B_{s_{1}}^{H} \cdots d B_{s_{n}}^{H},\right. \\
\left.F_{0} \in \mathbb{R}, f_{n} \in L^{2}\left(\mathbb{R}_{+}^{n}\right) \text { and } \sum_{n=1}^{\infty}\left|f_{n}\right|_{\phi}^{2}<\infty\right\}
\end{gathered}
$$

The equality (6.11) is described in the following theorem.

Theorem 6.9 If $F \in L^{2}(\Omega, \mathcal{F}, P)$, then there is a sequence $\left(f_{n} \in L_{\phi}^{2}\left(\mathbb{R}_{+}^{n}\right), n \in \mathbb{N}\right)$ such that $\sum_{n=1}^{\infty}\left|f_{n}\right|_{\phi}^{2}<\infty$ and

$$
F=\mathbb{E}(F)+\sum_{n=1}^{\infty} \int_{\mathbb{R}_{+}^{n}} f_{n}\left(s_{1}, \cdots, s_{n}\right) d B_{s_{1}}^{H} \cdots d B_{s_{n}}^{H} .
$$


Remark 4 The expansion (6.12) is an analogue of the Itô-Wiener chaos expansion which is extended to fractional Brownian motion. Replacing the multiple integrals by the iterated multiple integrals and summing the infinite series gives a stochastic integral representation for $F-\mathbb{E} F$. Note that the terms on the right hand side of (6.12) are orthogonal.

Remark 5 An earlier version of this paper was given to G. Kallianpur and he subsequently provided us with the preprint [3] where the multiple integrals of Stratonovich type are defined and their first and second moments are computed.

Remark 6 After the submission of this paper, the authors became aware of [5] where some related work on fractional Brownian motion is done.

\section{References}

[1] E.F. Beckenbach and R. Bellman Inequalities. Springer, 1965.

[2] W. Dai and C.C. Heyde, Itô formula with respect to fractional Brownian motion and its application, J. Appl. Math. Stoch. Anal. 9 (1996), 439-448.

[3] A. Dasgupta and G. Kallianpur, Multiple fractional integrals, Preprint, 1998.

[4] C. Dellacherie and P. A. Meyer, Probability and Potentials B, North Holland, 1982.

[5] L. Decreusefond and A.S. Üstünel, Stochastic analysis of the fractional Brownian motion, Potential Analysis, to appear.

[6] T.E. Duncan, Absolute continuity for abstract Wiener spaces, Pacific J. Math. 52 (1974), 359-367.

[7] G. Gripenberg and I. Norros, On the prediction of fractional Brownian motion, J. Appl. Prob. 33 (1996), 400-410.

[8] H. Holden, B. Øksendal, J. Ubøe and T.S. Zhang, Stochastic Partial Differential Equations, a Modeling, White Noise Functional Analysis, Birkhäuser, 1996.

[9] Y.Z. Hu and P.A.Meyer, Chaos de Wiener et intégrales de Feynman, in Séminaire de Probabilités XXII, ed. by J. Azema, P.A. Meyer and M. Yor, Lecture Notes in Mathematics 1321, Springer-Verlag, 1988, 51-71.

[10] Y.Z. Hu and P.A.Meyer, Sur les intégrales multiples de Stratonovich, in Séminaire de Probabilités XXVI, ed. by J. Azema, P.A. Meyer and M. Yor, Lecture Notes in Mathematics 1321, Springer-Verlag, 1988, 72-81.

[11] Y.Z. Hu and P.A.Meyer, On the approximation of Stratonovich multiple integrals, in Stochastic Processes, a festschrift in honor of G. Kallianpur, ed. by S. Cambanis, et al. 141-147, Springer, 1993. 
[12] Y.Z. Hu and B. Øksendal, Wick approximation of anticipating linear stochastic differential equations, in Stochastic Analysis and Related Topics, Progr. Prob. 38, Birkhäuser, Boston, 1996, 203-231.

[13] C. Houdré; V. Pérez-Abreu and A. S. Üstünel, Multiple Wiener-Itô integrals: an introductory survey. in Chaos expansions, multiple Wiener-Itô integrals and their applications (ed. by C. Houdré et al), 1-33, Probab. Stochastics Ser., CRC, Boca Raton, FL, 1994.

[14] H.E. Hurst, Long-term storage capacity in reservoirs, Trans. Amer. Soc. Civil Eng. 116 (1951), 400-410.

[15] H.E. Hurst, Methods of using long-term storage in reservoirs, Proc. Inst. Civil Engineers Part I, Chapter 5 (1956), 519-590.

[16] K. Itô, Multiple Wiener integrals, J. Math. Soc. Japan, 3 (1951), 157-164.

[17] S. J. Lin, Stochastic analysis of fractional Brownian motions, Stochastics Stochastics Rep. 55 (1995), 121-140.

[18] B.B. Mandelbrot, The Fractal Geometry of Nature. San Francisco, CA: Freeman, 1983.

[19] B.B. Mandelbrot and J.W. Van Ness, Fractional Brownian motions, fractional noises and applications, SIAM Rev. 10 (1968), 422-437.

[20] M. Métivier and J. Pellaumail, Stochastic Integration. Academic Press, New YorkLondon-Toronto, 1980.

[21] P.A. Meyer, Quantum probability for probabilists, Lect. Notes in Math. 1538, Springer, 1993.

[22] D. W. Stroock and S.R.S. Varadhan, Multidimensional Diffusion Processes, Springer, 1979.

[23] S. Watanabe, Stochastic Differential Equation and Malliavin Calculus, Tata Institute of Fundamental Research, Springer, 1984.

[24] N. Wiener, The homogeneous chaos, Amer. J. Math. 60 (1941), 897-936. 